TRANSACTIONS OF THE

AMERICAN MATHEMATICAL SOCIETY

Volume 362, Number 7, July 2010, Pages 3799-3826

S 0002-9947(10)05019-1

Article electronically published on February 24, 2010

\title{
HYPERBOLICITY OF GEOMETRIC ORBIFOLDS
}

\author{
ERWAN ROUSSEAU
}

\begin{abstract}
We study complex hyperbolicity in the setting of geometric orbifolds introduced by F. Campana. Generalizing classical methods to this context, we obtain degeneracy statements for entire curves with ramification in situations where no Second Main Theorem is known from value distribution theory.
\end{abstract}

\section{Contents}

1. Introduction

2. Geometric orbifolds, special varieties and hyperbolicity

2.1. Geometric orbifolds

2.2. Special varieties and hyperbolicity

3. Kobayashi hyperbolicity of orbifolds

3.1. Orbifold Kobayashi pseudo-distance

3.2. Hyperbolicity of orbifold curves

3.3. Hyperbolicity of higher-dimensional orbifolds

4. Algebraic hyperbolicity of orbifolds

4.1. The compact and the logarithmic setting

4.2. The orbifold case

5. An orbifold Kobayashi conjecture

6. Orbifold differential forms and applications

6.1. Symmetric differentials

6.2. Applications to hyperbolicity

7. Measure hyperbolicity and orbifolds of general type 3821

7.1. Kobayashi-Ochiai's extension theorems 3821

7.2. Orbifold measure hyperbolicity 3823

Acknowledgements $\quad 3824$

References

\section{INTRODUCTION}

F. Campana has introduced in [5] generalized orbifold structures, namely pairs $(X, \Delta)$ with $X$ a complex manifold and a divisor $\Delta=\sum_{i}\left(1-\frac{1}{m_{i}}\right) Z_{i}$, where the $Z_{i}$ are distinct irreducible divisors and $m_{i} \in \mathbb{N} \cup\{\infty\}$, as a new framework for the

Received by the editors September 15, 2008.

2000 Mathematics Subject Classification. Primary 14D06, 32H30, 32 Q45.

Key words and phrases. Orbifolds, Kobayashi hyperbolicity, entire curves.

(C)2010 American Mathematical Society Reverts to public domain 28 years from publication 
classification of compact Kähler manifolds. These structures appeared naturally for fibrations $f: X \rightarrow Y$. Indeed the multiple fibers of $f$ lead to the definition of the orbifold base of $f,(Y, \Delta(f))$, where

$$
\Delta(f):=\sum_{D \subset Y}\left(1-\frac{1}{m(f, D)}\right) D,
$$

$m(f, D)$ being the multiplicity (in the sense of Campana) of the fiber of $f$ above the generic point of $D$. A new class of varieties was then introduced, the special varieties, as the varieties which do not admit fibrations of general type, i.e., with an orbifold base of general type. Campana [5] proves the existence for every complex algebraic manifold $X$ of a fibration $c_{X}: X \rightarrow C(X)$, the core of $X$, such that its general fibers are special and if $X$ is not special, $c_{X}$ is of general type.

These geometric orbifolds should be considered as true geometric objects as one can define for them differential forms, fundamental groups, Kobayashi pseudodistance.... Here we study the hyperbolic aspects of these objects. An important conjecture of Campana [5] is that $X$ is special if and only if the Kobayashi pseudodistance $d_{X}$ vanishes identically on $X \times X$. This is known only for curves, projective surfaces not of general type and rationally connected manifolds.

This conjecture then implies that $d_{X}$ should be the pull-back by $c_{X}$ of the Kobayashi pseudo-distance $\delta_{X}$ of the orbifold base of the core.

The study of the hyperbolic aspects of one-dimensional orbifolds has been done in [8]. Here we study hyperbolicity of higher-dimensional orbifolds following the philosophy of Campana that one should study these objects by generalizing the tools we use for manifolds without orbifold structures or logarithmic manifolds. The paper is organized as follows.

In section 2, we recall the basic facts on geometric orbifolds following [5] and [6].

In section 3, we recall the definitions of classical and non-classical Kobayashi hyperbolicity for orbifolds. Then we illustrate these notions in the case of orbifold curves. We compute explicitly the non-classical orbifold Kobyashi pseudo-distance for

$$
(X, \Delta)=\left(\mathbb{D},\left(1-\frac{1}{n}\right)\{0\}\right), 0<n \in \mathbb{N} \cup\{\infty\},
$$

where $\mathbb{D}$ is the unit disk. This answers a question of Campana and Winkelmann (see remark, p. 20 of [8]) and enables us to recover as a corollary the equivalence of classical and non-classical hyperbolicity for orbifold curves (theorem 4 of [8]). Finally, we show that this is not the case in higher dimensions, giving an example of an orbifold surface which is classically hyperbolic but not hyperbolic.

In section 4 , we define and study algebraic hyperbolicity in the orbifold setting. We prove that $\left(\mathbb{P}^{n}, \Delta\right)$ is algebraically hyperbolic, where $\Delta=\sum_{1 \leq i \leq q}\left(1-\frac{1}{m_{i}}\right) H_{i}$ for $H_{1}, \ldots, H_{q}$ very generic hypersurfaces and $\operatorname{deg} \Delta>2 n$.

In section 5, we discuss an orbifold Kobayashi conjecture motivated by the results of the preceding section.

In section 6, we use orbifold symmetric differentials defined by Campana [5]. The main applications are algebraic degeneracy statements for entire curves with ramification in situations where no Second Main Theorem is known from value distribution theory. Namely, we prove

Theorem A. Let $(X, \Delta)$ be a smooth projective orbifold surface of general type (i.e., $X$ is a smooth projective surface, $\operatorname{supp}(\Delta)$ is a simple normal crossing divisor 
and $K_{X}+\Delta$ is big), where $\Delta$ has the following decomposition into irreducible components, $\Delta=\sum_{i=1}^{n}\left(1-\frac{1}{m_{i}}\right) C_{i}$. Suppose that $g_{i}:=g\left(C_{i}\right) \geq 2, h^{0}\left(C_{i}, \mathscr{O}_{C_{i}}\left(C_{i}\right)\right) \neq 0$ for all $i$ and that the logarithmic Chern classes of $(X,\lceil\Delta\rceil)$ satisfy

$$
{\overline{c_{1}}}^{2}-\overline{c_{2}}-\sum_{i=1}^{n} \frac{1}{m_{i}}\left(2 g_{i}-2+\sum_{j \neq i} C_{i} C_{j}\right)>0 .
$$

Then there exists a proper subvariety $Y \subsetneq X$ such that every entire curve $f: \mathbb{C} \rightarrow X$ which is an orbifold morphism, i.e., ramified over $C_{i}$ with multiplicity at least $m_{i}$, satisfies $f(\mathbb{C}) \subset Y$.

This result can be seen as an orbifold version of results of McQuillan [21] (see also 24] and [13] for the logarithmic case) on the Green-Griffiths-Lang conjecture, which can be generalized to the orbifold setting.

Conjecture B. Let $(X, \Delta)$ be a smooth projective orbifold of general type. Then there exists a proper subvariety $Y \subsetneq X$ such that every orbifold morphism $f: \mathbb{C} \rightarrow$ $(X, \Delta)$ satisfies $f(\mathbb{C}) \subset Y$.

The methods used also enable us to generalize a result of Campana and Paun on weakly-special manifolds 7 .

In section 7 , we study measure hyperbolicity of orbifolds.

\section{GeOMETRIC ORBIFOLDS, SPECIAL VARIETIES AND HYPERBOLICITY}

2.1. Geometric orbifolds. Let $X$ be a complex manifold. Following F. Campana (see [5, 6]) we recall the basic facts on the generalized orbifolds he introduced and why these structures should be useful to describe the hyperbolic aspects of projective manifolds.

Definition 2.1. A (geometric) orbifold $(X, \Delta)$ is a pair consisting of $X$ with a $\mathbb{Q}$-divisor $\Delta$ on $X$ for which the decomposition in reduced irreducible divisors is of the form

$$
\Delta=\sum_{i}\left(1-\frac{1}{m_{i}}\right) Z_{i}
$$

where $0<m_{i} \in \mathbb{N} \cup\{\infty\}$. (X, $\left.\Delta\right)$ is said to be smooth if $\operatorname{supp}(\Delta)$ is a simple normal crossing divisor.

Remark 2.2. We use the notion of orbifold in the sense of Campana. For the relation between this notion of geometric orbifold and the usual one (in the sense of Satake or Thurston) one may notice that one can attach to a usual orbifold $\mathscr{X}$ a geometric orbifold $(X, \Delta)$ with finite multiplicities $m_{i}$ (see for example [15]). Most pairs $(X, \Delta)$ are not obtained in this way. But if $(X, \Delta)$ is smooth, this is the case.

These structures appear naturally in the case of fibrations, i.e., surjective morphisms with connected fibres.

Definition 2.3. Let $f: X \rightarrow Y$ be a fibration between complex compact manifolds. For every irreducible divisor $D \subset Y$ we have

$$
f^{*}(D)=\sum_{j} m_{j} D_{j}+R
$$


where $R$ denotes the $f$-exceptional part; i.e., $f(R)$ is of codimension 2 at least. Then the multiplicity of $D$ is defined by

$$
m(f, D)=\inf _{j}\left\{m_{j}\right\}
$$

and the orbifold base $(Y, \Delta(f))$ is defined by

$$
\Delta(f)=\sum_{D \subset Y}\left(1-\frac{1}{m(f, D)}\right) D .
$$

Remark 2.4. These definitions are clearly unchanged if we change the birational model of $X$, and therefore these definitions make sense for meromorphic fibrations, i.e., surjective meromorphic maps whose desingularizations have connected fibers.

More generally, when $X$ is itself equipped with an orbifold structure, we have

Definition 2.5. Let $(X, \Delta)$ be an orbifold and $f:(X, \Delta) \rightarrow Y$ a fibration. For every irreducible divisor $D \subset Y$ we have

$$
f^{*}(D)=\sum_{j} m_{j} D_{j}+R
$$

where $R$ denotes the $f$-exceptional part. Then the multiplicity of $D$ is defined by

$$
m(f, \Delta, D)=\inf _{j}\left\{m_{j} \cdot m_{\Delta}\left(D_{j}\right)\right\},
$$

where $m_{\Delta}\left(D_{j}\right)$ is the multiplicity of $D_{j}$ in $\Delta$, and the orbifold base $(Y, \Delta(f))$ is defined by

$$
\Delta(f)=\sum_{D \subset Y}\left(1-\frac{1}{m(f, \Delta, D)}\right) D
$$

\section{Definition 2.6.}

(1) The canonical line bundle of $(X, \Delta)$ is defined by $K_{(X, \Delta)}:=K_{X}+\Delta$.

(2) The Kodaira dimension of $(X, \Delta)$ is $\kappa(X, \Delta):=\kappa\left(K_{(X, \Delta)}\right)$.

(3) Let $f: X \rightarrow Y$ be a fibration between complex compact manifolds. Then its Kodaira dimension is

$$
\kappa(f)=\inf _{f^{\prime} \sim f}\left\{\kappa\left(Y^{\prime}, \Delta\left(f^{\prime}\right)\right)\right\}
$$

where $f^{\prime}: X^{\prime} \rightarrow Y^{\prime}$ ranges over all holomorphic fibrations equivalent to $f$; i.e., there exists a commutative diagram

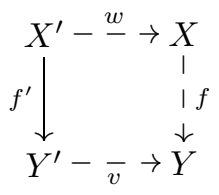

with $w$ and $v$ bimeromorphic.

Now, one can introduce the special geometry. 
2.2. Special varieties and hyperbolicity. Let $X, Y$ be projective manifolds.

\section{Definition 2.7.}

(1) A fibration $f: X \rightarrow Y$ is of general type if $\kappa(f)=\operatorname{dim} Y>0$.

(2) $X$ is special if there is no fibration $f: X \rightarrow Y$ of general type.

(3) $f: X \rightarrow Y$ is special if its general fiber is special.

Example 2.8. Rationally connected manifolds and manifolds with Kodaira dimension 0 are two important examples of special varieties (see [5]).

The following theorem makes clear why orbifold structures are useful for hyperbolicity.

Theorem 2.9 (Campana [5]). Let $X$ be a projective manifold. Then there exists a unique (up to equivalence) fibration

$$
c_{X}: X \rightarrow \mathscr{C}(X)
$$

called the core of $X$ such that

(1) $c_{X}$ is special.

(2) $c_{X}$ is of general type or constant (iff $X$ is special).

In other words, one can decompose a projective manifold into its "hyperbolic" part, the orbifold base of the core, and its "non-hyperbolic" part. Indeed, special varieties should be characterized by the following conjecture:

Conjecture 2.10 (Campana [5], p. 614). $X$ is special iff $d_{X} \equiv 0$, where $d_{X}$ denotes the Kobayashi pseudo-distance.

Remark 2.11. This is only known to be true for curves, projective surfaces not of general type, and rationally connected manifolds.

As a consequence, one should have the following description of the Kobayashi pseudo-distance:

Conjecture 2.12 (Campana [5, p. 617). $d_{X}=c_{X}^{*} \delta_{X}$ where $\delta_{X}$ is a pseudodistance on $\mathscr{C}(X)$.

We will make precise what that pseudo-distance $\delta_{X}$ should be in the next section.

\section{KOBAYASHI HYPERBOLICITY OF ORBIFOLDS}

3.1. Orbifold Kobayashi pseudo-distance. First, let us recall (following 8]) the definition of classical and non-classical orbifold morphisms from the unit disk to an orbifold.

Definition 3.1. Let $(X, \Delta)$ be an orbifold with $\Delta=\sum_{i}\left(1-\frac{1}{m_{i}}\right) Z_{i}, \mathbb{D}=\{z \in$ $\mathbb{C} /|z|<1\}$ the unit disk and $h$ a holomorphic map from $\mathbb{D}$ to $X$.

(1) $h$ is a (non-classical) orbifold morphism from $\mathbb{D}$ to $(X, \Delta)$ if $h(\mathbb{D}) \nsubseteq \operatorname{supp}(\Delta)$ and mult $_{x}\left(h^{*} Z_{i}\right) \geqslant m_{i}$ for all $i$ and $x \in \mathbb{D}$ with $h(x) \in \operatorname{supp}\left(Z_{i}\right)$. If $m_{i}=\infty$, we require $h(\mathbb{D}) \cap Z_{i}=\emptyset$.

(2) $h$ is a classical orbifold morphism from $\mathbb{D}$ to $(X, \Delta)$ if $h(\mathbb{D}) \nsubseteq \operatorname{supp}(\Delta)$ and mult $_{x}\left(h^{*} Z_{i}\right)$ is a multiple of $m_{i}$ for all $i$ and $x \in \mathbb{D}$ with $h(x) \in \operatorname{supp}\left(Z_{i}\right)$. If $m_{i}=\infty$, we require $h(\mathbb{D}) \cap Z_{i}=\emptyset$.

More generally we can define classical and non-classical orbifold morphisms between orbifolds. 
Definition $3.2\left([\underline{6})\right.$. Let $(X, \Delta)$ and $\left(X^{\prime}, \Delta^{\prime}\right)$ be orbifolds and $f: X \rightarrow X^{\prime}$ a holomorphic map. $f$ is an orbifold morphism (resp. classical orbifold morphism) from $(X, \Delta)$ to $\left(X^{\prime}, \Delta^{\prime}\right)$ if

(1) $f(X) \nsubseteq \operatorname{supp}\left(\Delta^{\prime}\right)$.

(2) For every irreducible divisor $D \subset X^{\prime}$ and $E \subset X$ such that $f^{*}(D)=$ $t_{E, D} \cdot E+R$, with $R$ an effective divisor of $X$ not containing $E$, we have

$t_{E, D} \cdot m_{X}(E) \geq m_{X^{\prime}}(D) \quad\left(\right.$ resp. $t_{E, D} \cdot m_{X}(E)$ is a multiple of $\left.m_{X^{\prime}}(D)\right)$,

where $m_{X}$ (resp. $m_{X^{\prime}}$ ) denotes the orbifold multiplicity on $X$ (resp. $X^{\prime}$ ).

Let $(X, \Delta)$ be an orbifold.

Definition 3.3. $\quad$ (1) The orbifold Kobayashi pseudo-distance $d_{(X, \Delta)}$ on $(X, \Delta)$ is the largest pseudo-distance on $X \backslash\lfloor\Delta\rfloor$ such that

$$
g^{*} d_{(X, \Delta)} \leqslant d_{P}
$$

for every orbifold morphism $g: \mathbb{D} \rightarrow(X, \Delta)$, where $d_{P}$ denotes the Poincaré distance on $\mathbb{D}$.

(2) The classical orbifold Kobayashi pseudo-distance $d_{(X, \Delta)}^{*}$ on $(X, \Delta)$ is the largest pseudo-distance on $X \backslash\lfloor\Delta\rfloor$ such that

$$
g^{*} d_{(X, \Delta)} \leqslant d_{P}
$$

for every classical orbifold morphism $g: \mathbb{D} \rightarrow(X, \Delta)$, where $d_{P}$ denotes the Poincaré distance on $\mathbb{D}$.

As an immediate consequence of the definition we have

Proposition 3.4. Let $f:(X, \Delta) \rightarrow\left(X^{\prime}, \Delta^{\prime}\right)$ be an orbifold morphism (resp. classical orbifold morphism). Then $f^{*} d_{\left(X^{\prime}, \Delta^{\prime}\right)} \leq d_{(X, \Delta)}\left(\right.$ resp. $\left.f^{*} d_{\left(X^{\prime}, \Delta^{\prime}\right)}^{*} \leq d_{(X, \Delta)}^{*}\right)$.

Definition 3.5. An orbifold $(X, \Delta)$ is hyperbolic (resp. classically hyperbolic) if $d_{(X, \Delta)}\left(\right.$ resp. $\left.d_{(X, \Delta)}^{*}\right)$ is a distance on $X \backslash\lfloor\Delta\rfloor$.

A corollary of Proposition 3.4 is

Corollary 3.6. Let $(X, \Delta)$ be a hyperbolic (resp. classically hyperbolic) orbifold. Then every orbifold morphism (resp. classical orbifold morphism) $f: \mathbb{C} \rightarrow(X, \Delta)$ is constant.

In the compact and logarithmic setting, Brody-type theorems turn out to be very useful to characterize hyperbolicity as a converse of Corollary [3.6. This is done in $[8$ with the following theorem.

Theorem 3.7. Let $(X, \Delta)$ be a non-hyperbolic (resp. non-classically hyperbolic) orbifold with $X$ compact. Then there exists either a non-constant orbifold morphism (resp. classical orbifold morphism) $f: \mathbb{C} \rightarrow(X, \Delta)$ or a non-constant holomorphic map $f: \mathbb{C} \rightarrow \operatorname{supp}(\Delta)$.

Then we can refine Conjecture 2.12

Conjecture 3.8 (Campana [5]). $d_{X}=c_{X}^{*} \delta_{X}$, where $\delta_{X}=d_{\left(\mathscr{C}(X), \Delta\left(c_{X}\right)\right)}$. 
3.2. Hyperbolicity of orbifold curves. Let us illustrate orbifold hyperbolicity in dimension 1 . This has been studied in [8]. Here we give a different approach. The following result gives a concrete example where one can compute the (non-classical) orbifold Kobayashi pseudo-distance, answering a question of [8].

Theorem 3.9. Let $(X, \Delta)=\left(\mathbb{D},\left(1-\frac{1}{n}\right)\{0\}\right), 0<n \in \mathbb{N} \cup\{\infty\}$. Then

$$
d_{(X, \Delta)}=d_{(X, \Delta)}^{*}
$$

is obtained as the pseudo-distance $d$ induced by

$$
\omega=\frac{4 d z d \bar{z}}{n^{2}|z|^{2-\frac{2}{n}}\left(1-|z|^{\frac{2}{n}}\right)^{2}} .
$$

Remark 3.10. For $n=\infty$ we obtain $\omega=\frac{4 d z d \bar{z}}{|z|^{2}\left(\log |z|^{2}\right)^{2}}$, which is known to induce the Kobayashi distance on the punctured disc.

To prove this we shall need the Ahlfors-Schwarz lemma (see, e.g., 11]).

Lemma 3.11 (Ahlfors-Schwarz lemma). Let $\gamma(t)=\gamma_{0}(t) d t d \bar{t}$ be a singular Hermitian metric on $\mathbb{D}$, where $\log \gamma_{0}$ is a subharmonic function such that $i \partial \bar{\partial} \log \gamma_{0}(t) \geq$ $A i \gamma_{0}(t) d t \wedge d \bar{t}$ in the sense of currents, for some positive constant $A$. Then

$$
\gamma(t) \leq \frac{2}{A} \frac{d t d \bar{t}}{\left(1-|z|^{2}\right)^{2}}=\frac{1}{2 A} h_{P}
$$

where $h_{P}$ denotes the Poincaré metric.

Now we prove the claim of the previous Theorem 3.9.

Proof. As $z \rightarrow z^{n}$ gives an unfolding $\mathbb{D} \rightarrow(X, \Delta)$ (see [8]), the classical Kobayashi pseudo-distance is the push-forward of the Poincaré metric on $\mathbb{D}$, which is

$$
\omega=\frac{4 d z d \bar{z}}{n^{2}|z|^{2-\frac{2}{n}}\left(1-|z|^{\frac{2}{n}}\right)^{2}} .
$$

Therefore we have $d_{(X, \Delta)} \leq d$.

Now, let $f: \mathbb{D} \rightarrow(X, \Delta)$ be an orbifold morphism. The observation is that $\omega$ defines a non-negatively curved metric on $K_{X}+\Delta$ and the condition that $f: \mathbb{D} \rightarrow$ $(X, \Delta)$ is an orbifold morphism will guarantee that $f^{*} \omega$ becomes a non-negatively curved (singular) metric on $K_{\mathbb{D}}$. Then we shall apply the above Ahlfors-Schwarz lemma to $f^{*} \omega$.

We have

$$
f^{*} \omega=\gamma_{0}(t) d t d \bar{t}:=\frac{4\left|f^{\prime}(t)\right|^{2}}{n^{2}|f(t)|^{2-\frac{2}{n}}\left(1-|f(t)|^{\frac{2}{n}}\right)^{2}} d t d \bar{t} .
$$

We remark that $\left(\frac{f^{\prime}(t)}{f(t)^{1-\frac{1}{n}}}\right)^{n}$ is holomorphic. Indeed take $t_{0}$ such that $f\left(t_{0}\right)=0$ and take a local coordinate $t$ centered at $t_{0}$. Then $f(t)=t^{m} g(t)$ with $m \geq n$ and $g(0) \neq 0$. Therefore $f^{\prime}(t)=t^{m-1} h(t)$ and

$$
\left(\frac{f^{\prime}(t)}{f(t)^{1-\frac{1}{n}}}\right)^{n}=\frac{t^{n(m-1)} h^{n}(t)}{t^{m(n-1)} g^{n-1}(t)}=t^{m-n} \frac{h^{n}(t)}{g^{n-1}(t)} .
$$

So $f^{*} \omega$ is a singular Hermitian metric with $\log \gamma_{0}$ subharmonic. Outside $f^{-1}(0)$ we have

$$
i \partial \bar{\partial} \log \gamma_{0}(t)=f^{*}(-\operatorname{Ricci}(\omega))=f^{*}\left(\frac{1}{2} \omega\right) .
$$


Therefore by the Ahlfors-Schwarz lemma,

$$
f^{*} \omega \leq h_{P}
$$

and finally $d_{(X, \Delta)} \geq d$.

Let us recall that the uniformization of orbifold curves is well known.

Theorem 3.12 (see 14, p. 234). Let $(M, \Delta)$ be an orbifold curve, with $M$ smooth, compact and

$$
\Delta:=\sum_{i}\left(1-\frac{1}{\nu_{i}}\right) x_{i}
$$

If $M=\mathbb{P}^{1}$ we exclude two cases:

(i) $\left\{x_{1}, x_{2}, \ldots\right\}$ consists of one point and $\nu_{1} \neq \infty$.

(ii) $\left\{x_{1}, x_{2}, \ldots\right\}$ consists of two points and $\nu_{1} \neq \nu_{2}$.

Let $M^{\prime}=M \backslash\lfloor\Delta\rfloor$. Then there exist a simply connected Riemann surface $\widetilde{M}$ and a Kleinian group $G$ of self-mappings of $\widetilde{M}$ such that

(a) $\widetilde{M} / G \cong M^{\prime}$;

(b) the natural projection $\pi: \widetilde{M} \rightarrow M^{\prime}$ is unramified except over the points $x_{k}$ with $\nu_{k}<\infty$ where the branch numbers satisfy $b_{\pi}(\widetilde{x})=\nu_{k}-1$ for all $\widetilde{x} \in \pi^{-1}\left(\left\{x_{k}\right\}\right)$.

Moreover,

(1) $\widetilde{M} \cong \mathbb{P}^{1}$ iff $\operatorname{deg}\left(K_{(M, \Delta)}\right)<0$,

(2) $\widetilde{M} \cong \mathbb{C}$ iff $\operatorname{deg}\left(K_{(M, \Delta)}\right)=0$,

(3) $\widetilde{M} \cong D$ iff $\operatorname{deg}\left(K_{(M, \Delta)}\right)>0$.

As a corollary we obtain

Corollary 3.13. Let $(X, \Delta)$ be a classically hyperbolic orbifold curve with $X$ compact. Then the classical and non-classical Kobayashi pseudo-distances coincide and therefore $(X, \Delta)$ is hyperbolic.

Proof. By Theorem 3.12 , $(X, \Delta)$ is uniformized by a Galois covering $\mathbb{D} \rightarrow(X, \Delta)$ which ramifies exactly on $\Delta$. So the classical Kobayashi pseudo-distance is induced by $\omega$, the push-forward of the Poincaré metric. Let $f: \mathbb{D} \rightarrow(X, \Delta)$ be an orbifold morphism. As the situation is locally the same as in Theorem 3.9] we obtain that $f^{*} \omega \leq h_{P}$ and therefore $d_{(X, \Delta)} \geq d_{(X, \Delta)}^{*}$.

Let us underline another property of hyperbolic orbifold curves which will be useful in the next section. In the case where $(M, \Delta)$ is uniformized by the unit disk $\mathbb{D}$, we can project the Poincaré metric $\frac{4|d z|^{2}}{\left(1-|z|^{2}\right)^{2}}$ on $M^{\prime}=M \backslash\lfloor\Delta\rfloor$, which gives a singular metric $h_{(M, \Delta)}$ inducing the Kobayashi pseudo-distance. Despite the singularities we have

Theorem 3.14 ([14, p. 233). The area of $M^{\prime}=M \backslash\lfloor\Delta\rfloor$ with respect to the metric $h_{(M, \Delta)}$ is finite and

$$
\operatorname{Area}\left(M^{\prime}\right)=2 \pi \operatorname{deg}\left(K_{(M, \Delta)}\right) .
$$

We have obtained a different proof of the result of 8$]$ where the authors prove the equivalence of classical and non-classical hyperbolicity for orbifolds of dimension 1 using the orbifold Brody's lemma and Nevanlinna theory. 
Theorem $3.15([8])$. Let $(X, \Delta)$ be an orbifold of dimension 1 with $X$ compact. Then the following properties are equivalent:

(1) $(X, \Delta)$ is hyperbolic;

(2) $(X, \Delta)$ is classically hyperbolic;

(3) $\operatorname{deg}\left(K_{(X, \Delta)}\right):=\operatorname{deg}\left(K_{X}+\Delta\right)>0$.

As a particular case, we recover the following classical result.

Theorem 3.16 (Nevanlinna [25]). Let $f: \mathbb{C} \rightarrow\left(\mathbb{P}^{1}, \sum_{i}\left(1-\frac{1}{m_{i}}\right) a_{i}\right)$ be a nonconstant orbifold morphism. Then

$$
\sum_{i}\left(1-\frac{1}{m_{i}}\right) \leq 2
$$

This theorem can be obtained thanks to the Second Main Theorem on $\mathbb{P}^{1}$. In section 6 of this paper, we will obtain similar results in higher dimension in cases where no Second Main Theorem is known.

3.3. Hyperbolicity of higher-dimensional orbifolds. Now we investigate the higher-dimensional case. In particular, is it still true that classical hyperbolicity coincides with (non-classical) hyperbolicity? The following example, found in a discussion with F. Campana, answers it in the negative:

Theorem 3.17. There exists an orbifold surface $(S, \Delta)$, with $S$ smooth and compact, which is classically hyperbolic but not hyperbolic.

Proof. Let $X$ be a hyperbolic projective complex surface, $S$ its blowup at one point $p$ and $E \subset S$ the exceptional divisor. We take $C_{1}, C_{2}, C_{3}$ to be three curves tangent to $E$ at three distinct points $p_{1}, p_{2}, p_{3}$. This can be done by taking the strict transforms of curves in $X$ with a cusp at $p$ of the local equation $(y+t x)^{2}-x^{3}=0$ for $t=0,1,-1$. Define

$$
\Delta=\left(1-\frac{1}{3}\right) C_{1}+\left(1-\frac{1}{3}\right) C_{2}+\left(1-\frac{1}{5}\right) C_{3} .
$$

Let us prove that $(S, \Delta)$ is classically hyperbolic but not hyperbolic.

The orbifold curve $\left(E, \Delta^{\prime}\right)$, where $\Delta^{\prime}$ is defined by

$$
\Delta^{\prime}=\left(1-\frac{1}{2}\right) p_{1}+\left(1-\frac{1}{2}\right) p_{2}+\left(1-\frac{1}{3}\right) p_{3},
$$

is not hyperbolic by Theorem 3.15 since

$$
-2+\left(1-\frac{1}{2}\right)+\left(1-\frac{1}{2}\right)+\left(1-\frac{1}{3}\right)=-\frac{1}{3}<0 .
$$

A non-constant orbifold morphism $f: \mathbb{C} \rightarrow\left(E, \Delta^{\prime}\right)$ gives a non-constant orbifold morphism $f: \mathbb{C} \rightarrow(S, \Delta)$ since the multiplicities satisfy respectively $2 \times 2 \geq$ $3,2 \times 2 \geq 3,2 \times 3 \geq 5$. Therefore $(S, \Delta)$ is not hyperbolic.

Suppose $(S, \Delta)$ is not classically hyperbolic. Then by Theorem 3.7 there is either a non-constant classical orbifold morphism $f: \mathbb{C} \rightarrow(S, \Delta)$ or a non-constant holomorphic map $f: \mathbb{C} \rightarrow \operatorname{supp}(\Delta)$. But since $X$ is hyperbolic we must have $f(\mathbb{C}) \subset E$. Therefore we obtain a non-constant classical orbifold morphism $f$ : $\mathbb{C} \rightarrow\left(E, \Delta^{\prime}\right)$ with

$$
\Delta^{\prime}=\left(1-\frac{1}{m_{1}}\right) p_{1}+\left(1-\frac{1}{m_{2}}\right) p_{2}+\left(1-\frac{1}{m_{3}}\right) p_{3},
$$


a divisor on $E$ such that the multiplicities satisfy, by the definition of a classical morphism, that $2 m_{1}$ and $2 m_{2}$ are multiples of 3 and $2 m_{3}$ is a multiple of 5 . So, $m_{1}$ and $m_{2}$ are multiples of $3, m_{3}$ is a multiple of 5 and we obtain

$-2+\left(1-\frac{1}{m_{1}}\right)+\left(1-\frac{1}{m_{2}}\right)+\left(1-\frac{1}{m_{3}}\right) \geq-2+\left(1-\frac{1}{3}\right)+\left(1-\frac{1}{3}\right)+\left(1-\frac{1}{5}\right)=\frac{2}{15}>0$.

This implies that $\left(E, \Delta^{\prime}\right)$ is hyperbolic and $f: \mathbb{C} \rightarrow\left(E, \Delta^{\prime}\right)$ should be constant, which is a contradiction. So, $(S, \Delta)$ is classically hyperbolic.

Now, we would like to define hyperbolic imbedding for orbifolds.

Definition 3.18. We say that $(X, \Delta)$ is hyperbolically (resp. classically hyperbolically) imbedded in $X$ if for any two sequences of points $\left(p_{n}\right),\left(q_{n}\right) \subset X \backslash\lfloor\Delta\rfloor$ converging to two points $p, q \in X$,

$$
d_{(X, \Delta)}\left(p_{n}, q_{n}\right) \underset{n \rightarrow+\infty}{\longrightarrow} 0 \Rightarrow p=q
$$

$\left(\right.$ resp. $\left.d_{(X, \Delta)}^{*}\left(p_{n}, q_{n}\right) \underset{n \rightarrow+\infty}{\longrightarrow} 0 \Rightarrow p=q\right)$.

This can be characterized by

Proposition 3.19. Let $\omega$ be a Hermitian metric on $X$ compact. Then $(X, \Delta)$ is hyperbolically (resp. classically hyperbolically) imbedded in $X$ iff there is a positive constant $c$ such that

$$
f^{*} \omega \leq c h_{P}
$$

for all orbifold (resp. classical orbifold) morphisms $f: \mathbb{D} \rightarrow(X, \Delta)$, where $h_{P}$ denotes the Poincaré metric.

Proof. Let us prove this in the non-classical case. If such a constant $c$ does not exist, then there exists a sequence $\left\{f_{n}\right\}$ of orbifold morphisms from $\mathbb{D}$ to $(X, \Delta)$ such that

$$
\left\|f_{n}^{\prime}(0)\right\|_{\omega}>n
$$

Since $X$ is compact we may assume that $\left\{f_{n}(0)\right\}$ converges to a point $p \in X$.

Let $U$ be a complete hyperbolic neighborhood of $p$ in $X$. Assume that there exists a positive number $r<1$ such that $f_{n}\left(\Delta_{r}\right) \subset U$ for $n \geq n_{0}$. Then $\left\{f_{n \mid \Delta_{r}}: \Delta_{r} \rightarrow U\right\}$ would be relatively compact and would have a subsequence which converges to a holomorphic function from $\Delta_{r}$ to $U$, which contradicts $\left\|f_{n}^{\prime}(0)\right\|_{\omega}>n$.

This means that for each positive integer $k$, there exist a point $z_{k} \in \Delta$ and an integer $n_{k}$ such that $\left|z_{k}\right|<\frac{1}{k}$ and $f_{n_{k}}\left(z_{k}\right) \notin U$. Let $p_{k}=f_{n_{k}}(0)$ and $q_{k}=f_{n_{k}}\left(z_{k}\right)$. By taking a subsequence we may assume that $\left\{q_{k}\right\}$ converges to a point $q$ not in $U$. Therefore we have

$$
d_{(X, \Delta)}\left(p_{k}, q_{k}\right) \leq d_{P}\left(0, z_{k}\right) \rightarrow 0 \text { for } k \rightarrow \infty,
$$

and this contradicts the fact that $(X, \Delta)$ is hyperbolically imbedded in $X$.

Conversely, let $\delta$ be the distance function on $X$ induced by $\frac{1}{c} \omega$. Then

$$
\delta \leq d_{(X, \Delta)}
$$

which implies obviously that $(X, \Delta)$ is hyperbolically imbedded in $X$. 


\section{Algebraic hyperbolicity of orbifolds}

4.1. The compact and the logarithmic setting. In [11], J.-P. Demailly introduced the concept of algebraic hyperbolicity for compact complex manifolds.

Definition 4.1. Let $X$ be a compact complex manifold and $\omega$ a Hermitian metric on $X . X$ is algebraically hyperbolic if there exists $\varepsilon>0$ such that every compact irreducible curve $C \subset X$ satisfies

$$
2 g(\widetilde{C})-2 \geq \varepsilon \operatorname{deg}_{\omega}(C),
$$

where $g(\widetilde{C})$ is the genus of the normalization $\widetilde{C}$ of $C$ and $\operatorname{deg}_{\omega}(C)=\int_{C} \omega$.

Later, algebraic hyperbolicity was defined in the logarithmic setting by X. Chen in [10, to which we refer for comments and motivations for the following definitions.

Definition 4.2. Let $X$ be a compact complex manifold, $\omega$ a Hermitian metric on $X$ and $D$ an effective reduced divisor on $X$. For each reduced irreducible curve $C \subset X$ such that $C \not \subset D$, let $\nu: \widetilde{C} \rightarrow C$ be the normalization of $C$. Then $i(C, D)$ is the number of distinct points in the set $\nu^{-1}(D) \subset \widetilde{C}$.

Definition 4.3. Let $X$ be a compact complex manifold, $\omega$ a Hermitian metric on $X$ and $D$ an effective reduced divisor on $X$. The logarithmic variety $(X, D)$ is algebraically hyperbolic if there exists a positive number $\varepsilon$ such that

$$
2 g(\widetilde{C})-2+i(C, D) \geq \varepsilon \operatorname{deg}_{\omega}(C)
$$

for all reduced and irreducible curves $C \subset X$ such that $C \not \subset D$, where $\widetilde{C}$ is the normalization of $C, g(\widetilde{C})$ its genus and $\operatorname{deg}_{\omega}(C)=\int_{C} \omega$ with $\omega$ a Hermitian metric on $X$.

4.2. The orbifold case. We give the following definition, which contains the two previous ones.

Definition 4.4. Let $(X, \Delta)$ be an orbifold with $X$ compact, $\omega$ a Hermitian metric on $X .(X, \Delta)$ is algebraically (resp. classically algebraically) hyperbolic if there exists $\varepsilon>0$ such that for any non-constant orbifold (resp. classical orbifold) morphism $f:\left(C, \Delta^{\prime}\right) \rightarrow(X, \Delta)$, where $\left(C, \Delta^{\prime}\right)$ is an orbifold curve with $C$ smooth,

$$
\operatorname{deg}\left(K_{\left(C, \Delta^{\prime}\right)}\right):=\operatorname{deg}\left(K_{C}+\Delta^{\prime}\right) \geq \varepsilon \int_{C} f^{*} \omega .
$$

Remark 4.5. Comparing Definition 4.3 and Definition 4.4 one can see, taking $(X, D)$ and $\nu: \widetilde{C} \rightarrow C$ as in Definition 4.3 that $\nu:\left(\widetilde{C}, \Delta^{\prime}\right) \rightarrow(X, \Delta)$ will be an orbifold morphism provided that $\Delta^{\prime} \geq \nu^{-1}(D)$. This shows the compatibility of the two definitions.

The interest of such a notion is to provide a necessary condition for analytic hyperbolicity which is more tractable since it involves only algebraic curves instead of transcendental ones. So the first thing to do is to prove that analytic hyperbolicity implies algebraic hyperbolicity. In the compact setting this was done in [11] and for the logarithmic case in 27. Here we will prove it in the more general setting of orbifolds.

Theorem 4.6. Let $(X, \Delta)$ be a hyperbolic (resp. classically hyperbolic) orbifold, with $X$ compact, hyperbolically imbedded in $X$. Then $(X, \Delta)$ is algebraically (resp. classically algebraically) hyperbolic. 
Proof. Let $f:\left(C, \Delta^{\prime}\right) \rightarrow(X, \Delta)$ be a non-constant orbifold morphism, where $\left(C, \Delta^{\prime}\right)$ is an orbifold curve. Since $(X, \Delta)$ is hyperbolic, $\left(C, \Delta^{\prime}\right)$ is hyperbolic. Therefore from Theorem 3.12 we obtain that there is an orbifold morphism $\pi$ : $\mathbb{D} \rightarrow\left(C, \Delta^{\prime}\right)$. As explained above this gives us a singular metric $h_{\left(C, \Delta^{\prime}\right)}$ on $C \backslash\left\lfloor\Delta^{\prime}\right\rfloor$ as the push-forward of the Poincaré metric by $\pi$. Moreover, since $(X, \Delta)$ is hyperbolically imbedded in $X$, we have $(f \circ \pi)^{*} \omega \leq c h_{P}$ with $c$ a positive constant. So, $f^{*} \omega \leq c h_{\left(C, \Delta^{\prime}\right)}$. Integrating and using Theorem 3.14, we obtain $\frac{1}{2 \pi c} \int_{C} f^{*} \omega \leq \operatorname{deg}\left(K_{\left(C, \Delta^{\prime}\right)}\right)$.

We shall generalize a result of J.-P. Demailly [1] stating that any morphism $f: A \rightarrow X$ from an abelian variety to a compact algebraically hyperbolic manifold is constant. We first recall some basic facts on semi-abelian varieties needed to state our result.

A quasiprojective variety $G$ is called a semi-abelian variety if it is a commutative group which admits an exact sequence of groups

$$
0 \rightarrow\left(\mathbb{C}^{*}\right)^{l} \rightarrow G \rightarrow A \rightarrow 0,
$$

where $A$ is an abelian variety.

We consider $G$ as a bundle over $A$ with fiber $\left(\mathbb{C}^{*}\right)^{l}$. Using the natural compactification $\left(\mathbb{C}^{*}\right)^{l} \subset\left(\mathbb{P}^{1}\right)^{l}$, we obtain a compactification $\bar{G}$ with boundary divisor $S$, which has only normal crossing singularities.

Theorem 4.7. Let $(X, \Delta)$ be an algebraically (resp. classically algebraically) hyperbolic orbifold with $X$ compact and $(\bar{G}, S)$ the logarithmic manifold associated to a semi-abelian variety $G$. Then any orbifold (resp. classically orbifold) morphism $f:(\bar{G}, S) \rightarrow(X, \Delta)$ is constant.

Proof. Let $C \subset G$ be a smooth curve with smooth compactification $\bar{C} \subset \bar{G}$. Let $m$ be a postive integer and consider the morphism $m_{G}: G \rightarrow G$ which is the multiplication by $m$ in $G$ and extends to a morphism $m_{\bar{G}}: \bar{G} \rightarrow \bar{G}$. We have the composition

$$
f_{m}: \bar{C} \subset \bar{G} \stackrel{m_{\bar{G}}}{\rightarrow} \bar{G} \stackrel{f}{\longrightarrow} X .
$$

Therefore we have an orbifold morphism

$$
f_{m}:\left(\bar{C}, \Delta^{\prime}\right) \rightarrow(X, \Delta),
$$

where $\Delta^{\prime}$ is the divisor induced on $\bar{C}$ by $S$. Let $L$ be an ample line bundle on $X$. We have

$$
\operatorname{deg}\left(K_{\bar{C}}+\Delta^{\prime}\right) \geq \varepsilon \bar{C} \cdot f_{m}^{*} L=\varepsilon m^{2} \bar{C} \cdot f^{*} L .
$$

As $m$ can be as large as we want, this shows that $\bar{C} \cdot f^{*} L=0$, so $f$ is constant on all curves in $G$ and therefore constant on $\bar{G}$.

An interesting example of an algebraically hyperbolic orbifold is given by the following theorem, which generalizes results of [10] and [27].

Theorem 4.8. Let $H_{1}, \ldots, H_{q}$ be very generic hypersurfaces of degrees $d_{i}$ in $\mathbb{P}^{n}$. Let

$$
\Delta=\sum_{1 \leq i \leq q}\left(1-\frac{1}{m_{i}}\right) H_{i} .
$$


Then for any non-constant orbifold morphism $f:(\widetilde{C}, \widetilde{\Delta}) \rightarrow(X, \Delta)$, where $(\widetilde{C}, \widetilde{\Delta})$ is an orbifold curve with $\widetilde{C}$ smooth,

$$
\operatorname{deg}\left(K_{(\widetilde{C}, \widetilde{\Delta})}\right) \geq(\operatorname{deg}(\Delta)-2 n) \operatorname{deg}(\widetilde{C}) .
$$

In particular if $\operatorname{deg}(\Delta)>2 n$, then $\left(\mathbb{P}^{n}, \Delta\right)$ is algebraically hyperbolic.

Proof. The proof will consist in a reduction to the logarithmic case (i.e., all mutiplicities are equal to $\infty$ ) where the result is known (see [10] and [27]). Let $D=\sum_{i=1}^{q} H_{i}$, $C \subset \mathbb{P}^{n}$ be a reduced irreducible curve not contained in $D$ and $f: \widetilde{C} \rightarrow C$ its normalization. Then it is sufficient to prove that

$$
\operatorname{deg}\left(K_{(\widetilde{C}, \widetilde{\Delta})}\right) \geq(\operatorname{deg}(\Delta)-2 n) \operatorname{deg}(C)
$$

for any orbifold $(\widetilde{C}, \widetilde{\Delta})$ such that $f:(\widetilde{C}, \widetilde{\Delta}) \rightarrow\left(\mathbb{P}^{n}, \Delta\right)$ is an orbifold morphism. First let us recall, as mentioned above, that in the logarithmic setting the result is known (see [10] and [27]), i.e.,

$$
2 g(\widetilde{C})-2+i(C, D) \geq(d-2 n) \operatorname{deg}(C),
$$

where $d=\sum_{i=1}^{q} d_{i}$. There is a minimal orbifold structure on $\widetilde{C}$ which makes $f$ an orbifold morphism, and it is of course sufficient to prove the result for this orbifold. Let

$$
\begin{aligned}
f^{*}\left(H_{j}\right) & =\sum_{i=1}^{i(C, D)} t_{i, j} p_{i}, \\
f^{*}(D) & =\sum_{i=1}^{i(C, D)} t_{i} p_{i},
\end{aligned}
$$

where the $p_{i}$ are the distinct points of $f^{-1}(D)$. Then if $\widetilde{\Delta}=\sum_{i=1}^{i(C, D)} m_{i}^{\prime} p_{i}$, the conditions for $f$ to be an orbifold morphism are $m_{i}^{\prime} t_{i} \geq m_{j}$ for all $j \in \varphi(i)=\{1 \leq$ $\left.k \leq i(C, D) / f\left(p_{i}\right) \in H_{k}\right\}$. Therefore the minimal orbifold structure is given by

$$
\widetilde{m_{i}}=\sup _{j \in \varphi(i)}\left\lceil\frac{m_{j}}{t_{i}}\right\rceil
$$

where $\lceil k\rceil$ denotes the rounding up of $k$. So we have to prove that

$$
2 g(\widetilde{C})-2+\sum_{i=1}^{i(C, D)}\left(1-\frac{1}{\widetilde{m_{i}}}\right) \geq(\operatorname{deg}(\Delta)-2 n) \operatorname{deg}(C) .
$$

We have

$$
\sum_{i=1}^{i(C, D)}\left(1-\frac{1}{\widetilde{m_{i}}}\right) \geq \sum_{i=1}^{i(C, D)}\left(1-\frac{t_{i}}{\sup _{j \in \varphi(i)} m_{j}}\right) \geq i(C, D)-\sum_{i=1}^{i(C, D)} \sum_{j=1}^{q} \frac{t_{i, j}}{m_{j}} .
$$

Moreover

Therefore

$$
\sum_{i=1}^{i(C, D)} t_{i, j}=\operatorname{deg}\left(f^{*} H_{j}\right)=C \cdot H_{j}=\operatorname{deg}(C) d_{j} .
$$

$$
\sum_{i=1}^{i(C, D)} \sum_{j=1}^{q} \frac{t_{i, j}}{m_{j}}=\operatorname{deg}(C)\left(\sum_{j=1}^{q} \frac{d_{j}}{m_{j}}\right) .
$$


From this inequality and Definition 4.1 we obtain

$$
2 g(\widetilde{C})-2+\sum_{i=1}^{i(C, D)}\left(1-\frac{1}{\widetilde{m}_{i}}\right) \geq(d-2 n) \operatorname{deg}(C)-\operatorname{deg}(C)\left(\sum_{j=1}^{q} \frac{d_{j}}{m_{j}}\right) .
$$

Finally,

$$
2 g(\widetilde{C})-2+\sum_{i=1}^{i(C, D)}\left(1-\frac{1}{\widetilde{m_{i}}}\right) \geq(\operatorname{deg}(\Delta)-2 n) \operatorname{deg}(C) .
$$

This example is a motivation for the conjecture introduced in the next section. As a corollary we obtain

Corollary 4.9. Let $H_{1}, \ldots, H_{q}$ be very generic hypersurfaces of degrees $d_{i}$ in $\mathbb{P}^{n}$. Let

$$
\Delta=\sum_{1 \leq i \leq q}\left(1-\frac{1}{m_{i}}\right) H_{i}
$$

such that $\operatorname{deg}(\Delta)>2 n$. Then every orbifold morphism $f: \mathbb{C} \rightarrow\left(\mathbb{P}^{n}, \Delta\right)$ whose image is contained in an algebraic curve is constant.

Proof. Let $f(\mathbb{C}) \subset C$. Then $f$ induces an orbifold morphism $f: \mathbb{C} \rightarrow\left(C^{\prime}, \Delta^{\prime}\right)$, where $C^{\prime}$ is the normalization of $C$ and $\Delta^{\prime}$ is the minimal orbifold structure making $\left(C^{\prime}, \Delta^{\prime}\right) \rightarrow\left(\mathbb{P}^{n}, \Delta\right)$ an orbifold morphism. But by the previous theorem we have in particular that $\left(C, \Delta^{\prime}\right)$ is hyperbolic. This implies that $f$ is constant.

\section{An orbifold Kobayashi COnJecture}

It is well known (see corollary 3.10.9, p. 137 of [18) that the complement of $2 n+1$ or more hyperplanes in general position in $\mathbb{P}^{n}$ is hyperbolic. More generally we shall prove

Theorem 5.1. Let $H_{1}, H_{2}, \ldots, H_{q}$ be $q$ hyperplanes in general position in $\mathbb{P}^{n}$ with $q>2 n$. Let $\Delta=\sum_{1 \leq i \leq q}\left(1-\frac{1}{m_{i}}\right) H_{i}$ with

$$
\operatorname{deg}(\Delta)>q-\frac{q}{n}+1+\frac{1}{n} .
$$

Then $\left(\mathbb{P}^{n}, \Delta\right)$ is hyperbolic and hyperbolically imbedded in $\mathbb{P}^{n}$.

We will prove this result using Nevanlinna theory, so let us recall the usual notation. Let $E=\sum_{i=1}^{\infty} \nu_{i} z_{i}$ be a divisor on $\mathbb{C}$ with distinct $z_{i} \in \mathbb{C}$. Then we define the counting functions of $E$ truncated to $l \leq \infty$ by

$$
\begin{aligned}
n_{l}(t, E) & =\sum_{\left\{\left|z_{i}\right|<t\right\}} \min \left\{\nu_{i}, l\right\}, \\
N^{l}(r, E) & =\int_{1}^{r} \frac{n_{l}(t, E)}{t} d t .
\end{aligned}
$$

If $D$ is a divisor on a complex space $X$ and $f: \mathbb{C} \rightarrow X$ is a holomorphic map not mapping to $D$, then

$$
N_{f}^{l}(r, D)=N^{l}\left(r, f^{*} D\right) .
$$

We denote $N_{f}(r, D):=N_{f}^{\infty}(r, D)$. 
If $\omega$ is a $(1,1)$-form on $X$, then the characteristic function with respect to $\omega$ is defined by

$$
T_{f}(r, \omega)=\int_{1}^{r}\left(\int_{|z|<t} f^{*} \omega\right) \frac{d t}{t} .
$$

If $L \rightarrow X$ is a line bundle on $X, h$ a metric on $L$ and $c_{h}$ the Chern form associated to $h$, then one can use $c_{h}$ to define the characteristic function corresponding to $L$. It is a classical result in Nevanlinna theory (see for example [31, p. 37) that the characteristic function is essentially independent of the choice of the metric and is determined not by the form but by the Chern class $c_{1}(L)$. Therefore we denote the characteristic function by $T_{f}\left(r, c_{1}(L)\right)$.

The defect is defined by

$$
\delta^{l}(f, D)=\liminf _{r \rightarrow \infty}\left(1-\frac{N_{f}^{l}(r, D)}{T_{f}\left(r, c_{1}(D)\right)}\right) .
$$

To state the result we need, let us recall the notion of hyperplanes in subgeneral position. Let $N \geq n$ and $q \geq N+1$. We consider $q$ hyperplanes $H_{1}, H_{2}, \ldots, H_{q}$ in $\mathbb{P}^{n}$, which are given by

$$
H_{j}:\left\langle Z, A_{j}\right\rangle=0
$$

for non-zero vectors $A_{j}$ in $\mathbb{C}^{n+1}$. Then we say that $H_{1}, H_{2}, \ldots, H_{q}$ are in $N$ subgeneral position if for every $1 \leq i_{0} \leq \cdots \leq i_{N} \leq q$,

$$
\operatorname{span}\left(A_{i_{0}}, \ldots, A_{i_{N}}\right)=\mathbb{C}^{n+1} .
$$

We have the following generalized defect relation of Cartan due to Nochka [26]:

Theorem 5.2. Let $H_{1}, H_{2}, \ldots, H_{q}$ be $q$ hyperplanes in $N$-subgeneral position in $\mathbb{P}^{n}$. Then for any holomorphic map $f: \mathbb{C} \rightarrow \mathbb{P}^{n}$ that is non-linearly degenerate, we have

$$
\sum_{i=1}^{q} \delta^{n}\left(f, H_{i}\right) \leq 2 N-n+1 .
$$

As a corollary we obtain

Theorem 5.3. Let $H_{1}, H_{2}, \ldots, H_{q}$ be $q$ hyperplanes in general position in $\mathbb{P}^{n}$ with $q>2 n$. Let $\Delta=\sum_{1 \leq i \leq q}\left(1-\frac{1}{m_{i}}\right) H_{i}$ with

$$
\operatorname{deg}(\Delta)>q-\frac{q}{n}+1+\frac{1}{n}
$$

Then every orbifold morphism $f: \mathbb{C} \rightarrow\left(\mathbb{P}^{n}, \Delta\right)$ is constant.

Proof. Suppose $\mathbb{P}^{l} \subset \mathbb{P}^{n}$ contains $f(\mathbb{C})$. The intersections of $\mathbb{P}^{l}$ with the $H_{i}$ are in $n$-subgeneral position in $\mathbb{P}^{l}$. By the First Main Theorem of Nevanlinna theory we have

$$
T_{f}\left(r, c_{1}\left(H_{i}\right)\right) \geq N_{f}\left(r, H_{i}\right)+C,
$$

where $C$ is a constant. Since $f^{*} H_{i}$ has multiplicity at least $m_{i}$ at every point of $f^{-1} H_{i}$ we have

$$
N_{f}\left(r, H_{i}\right) \geq \frac{m_{i}}{l} N_{f}^{l}\left(r, H_{i}\right)
$$

Therefore

$$
\delta^{l}\left(f, H_{i}\right) \geq 1-\frac{l}{m_{i}}
$$


Now

Therefore

$$
\sum_{i=1}^{q} \delta^{l}\left(f, H_{i}\right) \geq \sum_{i=1}^{q}\left(1-\frac{l}{m_{i}}\right)=l \operatorname{deg}(\Delta)-(l-1) q .
$$

$$
\sum_{i=1}^{q} \delta^{l}\left(f, H_{i}\right)>q\left(1-\frac{l}{n}\right)+l+\frac{l}{n} .
$$

From Theorem 5.2, we deduce that the conclusion holds provided that

$$
q\left(1-\frac{l}{n}\right)+l+\frac{l}{n} \geq 2 n-l+1
$$

for $l=1, \ldots, n$. This condition is clearly satisfied since

$$
q\left(1-\frac{l}{n}\right) \geq(2 n+1)\left(1-\frac{l}{n}\right)=2 n+1-2 l-\frac{l}{n} .
$$

Remark 5.4. We remark that corollary 3.10.8, p. 137 of $[18$ is a particular case of Theorem 5.3. namely the logarithmic one where all $m_{i}$ are equal to $\infty$.

Now, we can prove Theorem 5.1 .

Proof. Suppose $\left(\mathbb{P}^{n}, \Delta\right)$ is not hyperbolically imbedded in $\mathbb{P}^{n}$. We shall need a slight refinement of Theorem 3.7. There is a sequence of orbifold morphisms $f_{n}$ : $\mathbb{D} \rightarrow\left(\mathbb{P}^{n}, \Delta\right)$ such that $\lim \left\|f_{n}^{\prime}(0)\right\|=+\infty$. Thanks to Brody reparametrization, we obtain a sequence of orbifold morphisms $g_{n}: \mathbb{D}\left(0, r_{n}\right) \rightarrow\left(\mathbb{P}^{n}, \Delta\right)$, with $r_{n} \rightarrow+\infty$, converging to a holomorphic map $f: \mathbb{C} \rightarrow \mathbb{P}^{n}$ which is either a non-constant orbifold morphism $f: \mathbb{C} \rightarrow\left(\mathbb{P}^{n}, \Delta\right)$ or a non-constant holomorphic map $f: \mathbb{C} \rightarrow \operatorname{supp}(\Delta)$.

The first case is not possible thanks to Theorem 5.3 .

Consider a partition of indices $\{1,2, \ldots, q\}=I \cup J$, and let $L_{I}=\cap_{i \in I} H_{i}$. If $I$ contains $k$ elements, then $L_{I}$ is an $(n-k)$-dimensional linear subspace. The intersections $Z_{j}=H_{j} \cap L_{I}$ are $q-k$ hyperplanes in general position in $L_{I}$.

The observation here is that in the second case there is a partition $\{1,2, \ldots, q\}=$ $I \cup J$ such that $f$ is an orbifold morphism from $\mathbb{C}$ to $\left(L_{I}, \Delta^{\prime}\right)$, where $\Delta^{\prime}=$ $\sum_{i \in J}\left(1-\frac{1}{m_{i}}\right) Z_{i}$. Indeed, the sequence $g_{n}: \mathbb{D}\left(0, r_{n}\right) \rightarrow\left(\mathbb{P}^{n}, \Delta\right)$ can be seen as a sequence of orbifold morphisms $g_{n}: \mathbb{D}\left(0, r_{n}\right) \rightarrow\left(\mathbb{P}^{n}, \Delta_{J}\right)$, where $\Delta_{J}=$ $\sum_{i \in J}\left(1-\frac{1}{m_{i}}\right) H_{i}$ since $\Delta_{J} \leq \Delta$. Therefore (see [8]) it converges to a map $f$ which is either an orbifold morphism from $\mathbb{C}$ to $\left(\mathbb{P}^{n}, \Delta^{\prime}\right)$ or satisfies $f(\mathbb{C}) \subset \operatorname{supp}\left(\Delta^{\prime}\right)$.

Again using Theorem [5.3, we see that the conclusion holds provided that

$$
\begin{aligned}
q-k & >2(n-k), \\
\operatorname{deg}\left(\Delta_{J}\right) & >q-k-\frac{q-k}{n-k}+1+\frac{1}{n-k},
\end{aligned}
$$

for $k=1, \ldots, n-1$. The first condition is satisfied since $q>2 n$. For the second one, suppose it is not true. Then

$$
\operatorname{deg}(\Delta)=\operatorname{deg}\left(\Delta_{I}\right)+\operatorname{deg}\left(\Delta_{J}\right) \leq q-k-\frac{q-k}{n-k}+1+\frac{1}{n-k}+k .
$$

Therefore

$$
\operatorname{deg}(\Delta)-\left(q-\frac{q}{n}+1+\frac{1}{n}\right) \leq \frac{q-1}{n}-\frac{q-k-1}{n-k}=\frac{k(n+1-q)}{n(n-k)}<0 .
$$

So we obtain a contradiction. 
These results and Theorem 4.8 suggest the following generalization of a conjecture of S. Kobayashi (see [18, p. 78):

Conjecture 5.5. Let $H_{0}, H_{1}, \ldots, H_{q}$ be generic hypersurfaces in $\mathbb{P}^{n}$. Let

$$
\Delta=\sum_{0 \leq i \leq q}\left(1-\frac{1}{m_{i}}\right) H_{i},
$$

with $\operatorname{deg}(\Delta)>2 n$. Then $\left(\mathbb{P}^{n}, \Delta\right)$ is hyperbolic and hyperbolically imbedded in $\mathbb{P}^{n}$.

We will prove some more results towards this conjecture in the next section.

\section{ORBIFOLD DIFFERENTIAL FORMS AND APPLICATIONS}

6.1. Symmetric differentials. Let $(X, \Delta)$ be a smooth orbifold; i.e., the support of $\Delta, \operatorname{supp}(\Delta)$ (or $\lceil\Delta\rceil$ ), is a normal crossing divisor. Let $\left(x_{1}, \ldots, x_{n}\right)$ be local coordinates such that $\Delta$ has the equation

$$
x_{1}^{\left(1-\frac{1}{m_{1}}\right)} \ldots x_{n}^{\left(1-\frac{1}{m_{n}}\right)}=0 .
$$

Following [6] we can define sheaves of differential 1-forms on orbifolds.

Definition 6.1. For $N$ a positive integer, $S^{N} \Omega_{(X, \Delta)}^{1}$ is the locally free subsheaf of $S^{N} \Omega_{X}^{1}(\log \lceil\Delta\rceil)$ generated by the elements

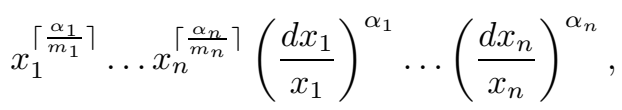

such that $\sum \alpha_{i}=N$, where $\lceil k\rceil$ denotes the round-up of $k$.

More generally, we can define orbifold $q$-forms.

Definition 6.2. For non-negative integers $N, q, S^{N} \Omega_{(X, \Delta)}^{q}$ is the locally free subsheaf of $S^{N} \Omega_{X}^{q}(\log \lceil\Delta\rceil)$ generated by the elements

$$
x^{\left\lceil\frac{k}{m}\right\rceil} \bigotimes_{l=1}^{l=N} \frac{d x_{J_{l}}}{x_{J_{l}}},
$$

where the $J_{l}$ are ordered sets of $q$ elements of $\{1, \ldots, n\}, x^{\left\lceil\frac{k}{m}\right\rceil}=\prod_{j=1}^{n} x_{j}^{\left\lceil\frac{k_{j}}{m_{j}}\right\rceil}$ for $k_{j}$ the number of times that $j$ appears in $J_{1}, \ldots, J_{N}$ and $\frac{d x_{J_{l}}}{x_{J_{l}}}=\bigwedge_{j \in J_{l}} \frac{d x_{j}}{x_{j}}$.

As an immediate consequence of the definition we have

Proposition $6.3\left([6)\right.$. Let $(X, \Delta)$ and $\left(Y, \Delta^{\prime}\right)$ be smooth orbifolds and $f:(X, \Delta) \rightarrow$ $\left(Y, \Delta^{\prime}\right)$ an orbifold morphism. Then

$$
f^{*}\left(S^{N} \Omega_{\left(Y, \Delta^{\prime}\right)}^{1}\right) \subset S^{N} \Omega_{(X, \Delta)}^{1} .
$$

6.2. Applications to hyperbolicity. It is well known in the compact and logarithmic cases that the existence of global symmetric differential forms vanishing on an ample divisor provides differential equations for entire curves. This can be generalized to the orbifold case as shown in [7].

Theorem 6.4 (lemma 4.10, [7). Let $(X, \Delta)$ be a smooth orbifold with $X$ compact, $A$ an ample line bundle on $X$ and $\omega \in H^{0}\left(X, S^{N} \Omega_{(X, \Delta)}^{1} \otimes A^{-1}\right)$. Then for any orbifold morphism $f: \mathbb{C} \rightarrow(X, \Delta)$,

$$
\omega\left(f^{\prime}\right) \equiv 0 .
$$


Here we would like to illustrate these ideas initiated in [7]. We provide some results towards Conjecture 5.5] which generalize [13] where the logarithmic case was studied.

Theorem 6.5. Let $(X, \Delta)$ be a smooth projective orbifold surface of general type, where $\Delta=\sum_{i=1}^{n}\left(1-\frac{1}{m_{i}}\right) C_{i}$ is the decomposition into irreducible components, and let $A$ be an ample line bundle on $X$. Suppose that $g_{i}:=g\left(C_{i}\right) \geq 2, h^{0}\left(C_{i}, \mathscr{O}_{C_{i}}\left(C_{i}\right)\right) \neq 0$ for all $i$ and that the logarithmic Chern classes of $(X,\lceil\Delta\rceil)$ satisfy

$$
{\overline{c_{1}}}^{2}-\overline{c_{2}}-\sum_{i=1}^{n} \frac{1}{m_{i}}\left(2 g_{i}-2+\sum_{j \neq i} C_{i} C_{j}\right)>0 .
$$

Then

for $N$ large enough.

$$
H^{0}\left(X, S^{N} \Omega_{(X, \Delta)}^{1} \otimes A^{-1}\right) \neq 0
$$

Proof. Let $F:=S^{N} \Omega_{X}^{1}(\log \lceil\Delta\rceil) / S^{N} \Omega_{(X, \Delta)}^{1}$ be the quotient sheaf. $F$ is supported on $C=\bigcup C_{i}$ and

$$
\begin{aligned}
h^{0}\left(X, S^{N} \Omega_{(X, \Delta)}^{1}\right) & \geq h^{0}\left(X, S^{N} \Omega_{X}^{1}(\log \lceil\Delta\rceil)\right)-h^{0}(C, F) \\
& \geq h^{0}\left(X, S^{N} \Omega_{X}^{1}(\log \lceil\Delta\rceil)\right)-\sum_{i=1}^{n} h^{0}\left(C_{i}, F_{\mid C_{i}}\right) .
\end{aligned}
$$

$F_{\mid C_{i}}$ has a natural filtration

$$
F_{N} \subset F_{N-1} \subset \cdots \subset F_{1} \subset F_{0}=F_{\mid C_{1}},
$$

such that

$$
F_{j} / F_{j+1}=S^{N-j} \Omega_{\left(C_{i}, \Delta_{i}\right)}^{1} \otimes G_{j},
$$

where $\Delta_{i}:=\sum_{j \neq i}\left(1-\frac{1}{m_{j}}\right) \mathscr{O}_{C_{i}}\left(C_{j}\right)$ is the divisor induced by $\Delta$ on $C_{i}$ and $G_{j}$ is a locally free sheaf of rank $r_{j}:=\left\lceil\frac{j}{m_{i}}\right\rceil$ admitting a filtration

$$
H_{1} \subset H_{1} \subset \cdots \subset H_{r_{j}}=G_{j}
$$

where

$$
H_{i} / H_{i-1}=\left(\mathscr{N}_{C_{i}}^{*}\right)^{\otimes i}
$$

with $\mathscr{N}_{C_{i}}$ denoting the normal bundle of $C_{i}$. We have

$$
S^{N-j} \Omega_{\left(C_{i}, \Delta_{i}\right)}^{1}=\left\lfloor(N-j)\left(K_{C_{i}}+\Delta_{i}\right)\right\rfloor .
$$

Since $\mathscr{N}_{C_{i}}=\mathscr{O}_{C_{i}}\left(C_{i}\right)$ and $h^{0}\left(C_{i}, \mathscr{O}_{C_{i}}\left(C_{i}\right)\right) \neq 0$ we obtain

$$
\begin{aligned}
h^{0}\left(C_{i}, F_{\mid C_{i}}\right) \leq & \sum_{j=0}^{N} h^{0}\left(C_{i}, F_{j} / F_{j+1}\right) \\
\leq & h^{0}\left(C_{i}, N\left(K_{C_{i}}+\sum_{j \neq i} \mathscr{O}_{C_{i}}\left(C_{j}\right)\right)\right. \\
& +\sum_{j=1}^{N} r_{j} h^{0}\left(C_{i},(N-j)\left(K_{C_{i}}+\sum_{j \neq i} \mathscr{O}_{C_{i}}\left(C_{j}\right)\right) .\right.
\end{aligned}
$$

Since $g_{i} \geq 2$ we obtain for $N-j \geq 1$,

$$
h^{0}\left(C_{i},(N-j)\left(K_{C_{i}}+\sum_{j \neq i} \mathscr{O}_{C_{i}}\left(C_{j}\right)\right) \leq(N-j)\left(2 g_{i}-2+\sum_{j \neq i} C_{i} C_{j}\right)-g_{i}+1\right.
$$


Now we suppose that $N=q m_{i}$ is a multiple of $m_{i}$. We write $j=(h-1) m_{i}+k$ with $1 \leq k \leq m_{i}$, so $r_{j}:=h$. Therefore we obtain

$$
\begin{aligned}
h^{0}\left(C_{i}, F_{\mid C_{i}}\right) \leq & m_{i} q\left(2 g_{i}-2+\sum_{j \neq i} C_{i} C_{j}\right)-g_{i}+1 \\
& +\sum_{h=1}^{q} m_{i}\left(h m_{i}(q-h+1)\left(2 g_{i}-2+\sum_{j \neq i} C_{i} C_{j}\right)-g_{i}+1\right) \\
\leq & \frac{1}{6} m_{i}^{2} q^{3}\left(2 g_{i}-2+\sum_{j \neq i} C_{i} C_{j}\right)+O\left(q^{2}\right) .
\end{aligned}
$$

Therefore we obtain if $N=q m_{1} \ldots m_{n}$,

$$
\sum_{i=1}^{n} h^{0}\left(C_{i}, F_{\mid C_{i}}\right) \leq \frac{1}{6}\left(m_{1} \ldots m_{n} q\right)^{3}\left(\sum_{i=1}^{n} \frac{1}{m_{i}}\left(2 g_{i}-2+\sum_{j \neq i} C_{i} C_{j}\right)\right)+O\left(q^{2}\right) .
$$

On the other hand,

$$
\chi\left(X, S^{N} \Omega_{X}(\log \lceil\Delta\rceil)\right)=\frac{\left(m_{1} \ldots m_{n} q\right)^{3}}{6}\left({\overline{c_{1}}}^{2}-\overline{c_{2}}\right)+O\left(q^{2}\right) .
$$

Using Bogomolov's vanishing theorem [2] for the $h^{2}$ term we obtain

$$
h^{0}\left(X, S^{N} \Omega_{X}^{1}(\log \lceil\Delta\rceil)\right) \geq \frac{\left(m_{1} \ldots m_{n} q\right)^{3}}{6}\left({\overline{c_{1}}}^{2}-\overline{c_{2}}\right)+O\left(q^{2}\right) .
$$

So we have

$$
h^{0}\left(X, S^{N} \Omega_{(X, \Delta)}^{1}\right) \geq \frac{\left(m_{1} \ldots m_{n} q\right)^{3}}{6}\left({\overline{c_{1}}}^{2}-\overline{c_{2}}-\sum_{i=1}^{n} \frac{1}{m_{i}}\left(2 g_{i}-2+\sum_{j \neq i} C_{i} C_{j}\right)\right)+O\left(q^{2}\right) .
$$

Finally, take a (very) ample line bundle $A$ and consider the exact sequence

$$
0 \rightarrow S^{N} \Omega_{(X, \Delta)}^{1} \otimes A^{-1} \rightarrow S^{N} \Omega_{(X, \Delta)}^{1} \rightarrow S^{N} \Omega_{(X, \Delta)}^{1} \mid A \rightarrow 0 .
$$

Then the growth of $h^{0}\left(X, S^{N} \Omega_{(X, \Delta)}^{1}\right)$ just obtained, combined with the fact that $h^{0}\left(A, S^{N} \Omega_{(X, \Delta)}^{1} \mid A\right)=O\left(q^{2}\right)$, gives non-zero global sections of $S^{N} \Omega_{(X, \Delta)}^{1} \otimes A^{-1}$.

As a first application, we can generalize a result due to Bogomolov [1] about the finiteness of rational and elliptic curves on surfaces of general type with $c_{1}^{2}>c_{2}$.

Theorem 6.6. Let $(X, \Delta)$ be a smooth projective orbifold surface of general type with the same hypotheses as in Theorem 6.5. Then there are only finitely many special curves $C \subset(X, \Delta)$, i.e., images of non-constant orbifold morphisms $\nu$ : $\left(C^{\prime}, \Delta^{\prime}\right) \rightarrow(X, \Delta)$, where $\left(C^{\prime}, \Delta^{\prime}\right)$ is an orbifold curve with $\operatorname{deg}\left(K_{\left(C^{\prime}, \Delta^{\prime}\right)} \leq 0\right.$.

Proof. Let $Y=\mathbb{P}\left(T_{X}(-\log [\Delta\rceil)\right)$ be the projectivization of the logarithmic tangent bundle. From Theorem 6.5 there is a global section $\omega \in H^{0}\left(X, S^{N} \Omega_{(X, \Delta)} \otimes A^{-1}\right)$ which can be seen as a holomorphic section of $\mathscr{O}_{Y}(N) \otimes \pi^{*} A^{-1}$, where $\pi: Y \rightarrow X$ denotes the canonical projection. Now, from Theorem 6.4 the lifts of the special curves must lie in an irreducible component $Z \subset Y$ of the zeros of $\omega$. Let $V \subset$ $T_{Y}\left(-\log \pi^{*}\lceil\Delta\rceil\right)$ be the subbundle defined by

$$
V_{x,[v]}=\left\{\xi \in T_{Y}\left(-\log \pi^{*}\lceil\Delta\rceil\right) \mid(\pi)_{*} \xi \in \mathbb{C} . v\right\} .
$$

Then $V$ defines on the desingularization $\widetilde{Z}$ of $Z$ a foliation by curves, such that the tangent bundle to the leaves is given by $T_{Z} \cap V$. The lifts of the special curves are 
leaves of this foliation. Now, a theorem of Jouanolou [17] implies that if there is an infinite number of such curves, then there is a meromorphic fibration $\widetilde{Z} \rightarrow S$ from $\widetilde{Z}$ to a curve such that the leaves correspond to the fibers of the fibration. Let $\widetilde{\Delta} \subset \widetilde{Z}$ be the divisor above $\Delta$. Then $(\widetilde{Z} / \widetilde{\Delta})$ is of general type. But we cannot have a fibration $(\widetilde{Z} / \widetilde{\Delta}) \rightarrow S$ with special generic fiber $(\widetilde{Z} / \widetilde{\Delta})_{s}$ and at the same time $(\widetilde{Z} / \widetilde{\Delta})$ of general type (see corollary 7.14 of $[6]$ ).

Now, we turn to the transcendental case. We will use the following result, which is a consequence of deep works of McQuillan on foliations of surfaces (see 21, 23], 24 and also 13 for a weaker version). For the convenience of the reader we will give a proof, referring to the above-mentioned articles for details.

Theorem 6.7. Let $(X, D)$ be a smooth logarithmic projective surface of log general type (i.e. $X$ is a smooth projective surface, $D$ a simple normal crossing divisor such that $K_{X}+D$ is big) and let $f: \mathbb{C} \rightarrow X$ be an algebraically non-degenerate entire curve. Let $f_{[1]}: \mathbb{C} \rightarrow \mathbb{P}\left(T_{X}(-\log D)\right)$ denote the canonical lifting of $f$. Suppose $f_{[1]}(\mathbb{C})$ is contained in a divisor in $\mathbb{P}\left(T_{X}(-\log D)\right)$. Then

$$
T_{f}\left(r, c_{1}\left(K_{X}+D\right)\right) \leq N_{f}^{1}(r, D)+\epsilon T_{f}\left(r, c_{1}(H)\right) \|_{\epsilon}
$$

for some ample line bundle $H$ on $X$ and the notation $\|_{\epsilon}$ meaning that the inequality holds for any $\epsilon>0$, for $r$ outside a subset of finite measure depending on $\epsilon$.

Proof. Let $S \subset \mathbb{P}\left(T_{X}(-\log D)\right)$ denote the surface which contains $f(\mathbb{C})$ and $\pi$ : $S \rightarrow X$ the canonical projection. As already explained in the proof of Theorem 6.6, there is a canonical foliation $\mathscr{F}$ on $S$ such that $f_{[1]}: \mathbb{C} \rightarrow \mathbb{P}\left(T_{X}(-\log D)\right)$ is a leaf of $\mathscr{F}$. After some blow ups we obtain a foliated smooth surface $(\widetilde{S}, \widetilde{D}, \widetilde{F}) \rightarrow$ $\left(S, \pi^{-1}(D), \mathscr{F}\right)$; i.e., $\widetilde{S}$ is smooth, $\widetilde{D}$ is normal crossing and $\widetilde{\mathscr{F}}$ has reduced singularities thanks to 30 . Let $\widetilde{D}=C+B$, where $C$ is the invariant part of $\widetilde{D}$ by $\widetilde{\mathscr{F}}$. We have an exact sequence

$$
0 \rightarrow \mathscr{N}^{*}(C) \rightarrow T_{\widetilde{S}}^{*}(\log \widetilde{D}) \rightarrow K_{\widetilde{F}}(B) . \mathscr{I}_{Z} \rightarrow 0,
$$

where $\mathscr{I}_{Z}$ is an ideal supported on the singularity set $Z$ of $\mathscr{F}$. Now, we apply the logarithmic tautological inequality of McQuillan (see [22] and [33]) which gives

$$
T_{\widetilde{f}_{[1]}}\left(r, c_{1}(L)\right) \leq N_{\widetilde{f}}^{1}(r, \widetilde{D})+\epsilon T_{f}\left(r, c_{1}(H)\right) \|_{\epsilon},
$$

where $L=\mathscr{O}_{\mathbb{P}\left(T_{\tilde{S}}(-\log \tilde{D})\right)}(1)$ and $\widetilde{f}$ and $\widetilde{f}_{[1]}$ are the lifts of $f$. Moreover, we have

$$
L_{\mid Y}=p^{*} K_{\widetilde{F}}(B) \otimes \mathscr{O}(-E),
$$

where $L_{\mid Y}$ denotes the restriction of $L$ to the graph $Y$ of the foliation, $p: Y \rightarrow \widetilde{S}$ the projection and $E$ is the total exceptional divisor. Therefore we obtain

$$
T_{\widetilde{f}}\left(r, c_{1}\left(K_{\widetilde{F}}+B\right)\right) \leq N_{\widetilde{f}}^{1}(r, \widetilde{D})+\epsilon T_{f}\left(r, c_{1}(H)\right) \|_{\epsilon} .
$$

Indeed, it is proved in 21] (see also [23, 24, 4] and 13]), even if it means doing some more blow ups on $\widetilde{S}$ and working on this new surface, that $T_{\widetilde{f}_{[1]}}\left(r, c_{1}(E)\right) \leq$ $\epsilon T_{f}\left(r, c_{1}(H)\right) \|_{\epsilon}$. It is also proved (see [21], [23], [24, [4] and [13]) that 
$T_{\widetilde{f}}\left(r, c_{1}\left(\mathscr{N}^{*}(C)\right)\right) \leq \epsilon T_{f}\left(r, c_{1}(H)\right) \|_{\epsilon}$. Therefore we obtain

$$
T_{\widetilde{f}}\left(r, c_{1}\left(K_{\widetilde{S}}+\widetilde{D}\right)\right) \leq N_{\widetilde{f}}^{1}(r, \widetilde{D})+\epsilon T_{f}\left(r, c_{1}(H)\right) \|_{\epsilon},
$$

and finally

$$
T_{f}\left(r, c_{1}\left(K_{X}+D\right)\right) \leq N_{f}^{1}(r, D)+\epsilon T_{f}\left(r, c_{1}(H)\right) \|_{\epsilon} .
$$

Now, we can prove

Theorem 6.8. Let $(X, \Delta)$ be a smooth projective orbifold surface of general type with the same hypotheses as in Theorem 6.5. Then every orbifold morphism $f$ : $\mathbb{C} \rightarrow(X, \Delta)$ is algebraically degenerate.

Proof. Suppose $f: \mathbb{C} \rightarrow(X, \Delta)$ is algebraically non-degenerate. Let $D:=\lceil\Delta\rceil$. By Theorems 6.5] and 6.4, $f_{[1]}(\mathbb{C})$ is contained in a divisor in $\mathbb{P}\left(T_{X}(-\log D)\right)$. Then we can apply Theorem 6.7 and we have

$$
T_{f}\left(r, c_{1}\left(K_{X}+D\right)\right) \leq N_{f}^{1}(r, D)+\epsilon T_{f}\left(r, c_{1}(H)\right) \|_{\epsilon},
$$

where $H$ is an ample divisor. Moreover

$$
T_{f}\left(r, c_{1}\left(K_{X}+\Delta\right)\right)=T_{f}\left(r, c_{1}\left(K_{X}\right)\right)+\sum_{i=1}^{n}\left(1-\frac{1}{m_{i}}\right) T_{f}\left(r, c_{1}\left(C_{i}\right)\right) .
$$

We have $m_{i} N_{f}^{1}\left(r, C_{i}\right) \leq N_{f}\left(r, C_{i}\right)$ and, by the First Main Theorem, $N_{f}\left(r, C_{i}\right) \leq$ $T_{f}\left(r, c_{1}\left(C_{i}\right)\right)+O(1)$. Therefore

$$
T_{f}\left(r, c_{1}\left(C_{i}\right)\right)-N_{f}^{1}\left(r, C_{i}\right) \geq\left(1-\frac{1}{m_{i}}\right) T_{f}\left(r, c_{1}\left(C_{i}\right)\right)+O(1) .
$$

So we obtain

$$
T_{f}\left(r, c_{1}\left(K_{X}+\Delta\right)\right) \leq T_{f}\left(r, c_{1}\left(K_{X}\right)\right)+\sum_{i=1}^{n}\left(T_{f}\left(r, c_{1}\left(C_{i}\right)\right)-N_{f}^{1}\left(r, C_{i}\right)\right)+O(1) .
$$

Also,

$$
T_{f}\left(r, c_{1}\left(K_{X}+\Delta\right)\right) \leq T_{f}\left(r, c_{1}\left(K_{X}+D\right)\right)-N_{f}^{1}(r, D)+O(1),
$$

which gives

$$
T_{f}\left(r, c_{1}\left(K_{X}+\Delta\right)\right) \leq \epsilon T_{f}\left(r, c_{1}(H)\right) \|_{\epsilon} .
$$

We shall deduce a contradiction from the fact that $(X, \Delta)$ is of general type. Indeed, write $K_{X}+\Delta=\frac{1}{m}(H+E)$, where $E$ is an effective divisor and $m$ a sufficiently large integer.

Then we obtain, using the First Main Theorem $T_{f}\left(r, c_{1}(E)\right) \geq N_{f}(r, E)+O(1)$, that

$$
\frac{1}{m} T_{f}\left(r, c_{1}(H)\right)+O(1) \leq \epsilon T_{f}\left(r, c_{1}(H)\right) \|_{\epsilon} .
$$

Since $T_{f}\left(r, c_{1}(H)\right) \rightarrow+\infty$ as $r \rightarrow+\infty$, this gives a contradiction.

Therefore $f$ is algebraically degenerate.

Remark 6.9. These results generalize [13, where all multiplicities are infinite. 
As a consequence of Theorems 6.6 and 6.8, we obtain the proof of

Theorem A. Let $(X, \Delta)$ be a smooth compact orbifold surface of general type with the same hypotheses as in Theorem 6.5. Then there exists a proper subvariety $Y \subsetneq X$ such that every orbifold morphism $f: \mathbb{C} \rightarrow(X, \Delta)$ satisfies $f(\mathbb{C}) \subset Y$.

6.2.1. Degeneracy of holomorphic curves with ramification. As an application we obtain the following theorem.

Theorem 6.10. Let $C_{i}, 1 \leq i \leq 2$, be two smooth curves in $X=\mathbb{P}^{2}$ of degree $d_{i} \geq 4$ with normal crossings. Let $\Delta=\left(1-\frac{1}{m_{1}}\right) C_{1}+\left(1-\frac{1}{m_{2}}\right) C_{2}$, and $d=d_{1}+d_{2}$. If

$$
\operatorname{deg}(\Delta)>\frac{d_{1}^{2}+d_{2}^{2}+d_{1} d_{2}-6}{d-3}
$$

then every orbifold morphism $f: \mathbb{C} \rightarrow(X, \Delta)$ is algebraically degenerate. Moreover, if the curves $C_{i}$ are very generic, then $(X, \Delta)$ is hyperbolic.

Proof. First we verify that condition (6.1) is satisfied. We compute everything in terms of the degrees $d_{1} \leq d_{2}$ :

$$
\begin{aligned}
{\overline{c_{1}}}^{2}-\overline{c_{2}}-\frac{1}{m_{1}}\left(2 g_{1}-2+d_{1} d_{2}\right)-\frac{1}{m_{2}}\left(2 g_{2}-2+d_{1} d_{2}\right) \\
=\operatorname{deg}(\Delta)(d-3)-\left(d_{1}^{2}+d_{2}^{2}+d_{1} d_{2}-6\right) .
\end{aligned}
$$

So, if condition (6.2) is satisfied, we can apply Theorem $\mathrm{A}$ and obtain the algebraic degeneracy of $f$. If ${\overline{c_{1}}}^{2}-\overline{c_{2}}>0$, then $d_{1} \geq 5$ or $d_{1} \geq 4$ and $d_{2} \geq 7$ (see [28]). Therefore $\operatorname{deg}(\Delta)>4$ and, if the curves are very generic, from Corollary 4.9 we obtain that $f$ is constant.

Example 6.11. Let $C_{i}, 1 \leq i \leq 2$ be two smooth curves in $\mathbb{P}^{2}$ of degree 5 with normal crossings. Let $\Delta=\left(1-\frac{1}{70}\right) C_{1}+\left(1-\frac{1}{71}\right) C_{2}$. Then every orbifold morphism $f: \mathbb{C} \rightarrow(X, \Delta)$ is algebraically degenerate. If the curves $C_{i}$ are very generic, then $\left(\mathbb{P}^{2}, \Delta\right)$ is hyperbolic.

6.2.2. Weakly-special manifolds with degenerate entire curves. Let us recall that a complex projective manifold is said to be weakly-special if none of its finite etale covers has a dominant rational map to a positive-dimensional manifold of general type. Bogomolov and Tschinkel constructed in [3] examples of algebraic threefolds which are weakly-special but non-special. These examples are simply connected and come with an elliptic fibration $\varphi: X \rightarrow B$, where $B$ is a surface with $\kappa(B)=1$. This fibration is of general type because it has multiple fibres of multiplicity $m \geq 2$ over a smooth curve $D$ such that $\kappa\left(B, K_{B}+\left(1-\frac{1}{m}\right) D\right)=2$. But these multiple fibers cannot be eliminated by an etale cover of $X$, which is simply connected. We remark that our results enable us to simplify the proof and generalize the results of [7] where it is proved that in some of the examples of [3] all entire curves are degenerate. Indeed, using this notation, an immediate consequence of Theorem A is

Theorem 6.12. Let $X$ be a Bogomolov-Tschinkel example. If

$$
{\overline{c_{1}}}^{2}(B, D)-\overline{c_{2}}(B, D)-\frac{1}{m}(2 g(D)-2)>0,
$$

then there exists $\Gamma \subsetneq B$ such that for any entire curve $h: \mathbb{C} \rightarrow X, \varphi \circ h: \mathbb{C} \rightarrow B$ is either a point or contained in $\Gamma$. 
Proof. We just remark that $\varphi \circ h: \mathbb{C} \rightarrow\left(B,\left(1-\frac{1}{m}\right) D\right)$ is an orbifold morphism and apply Theorem A.

Remark 6.13. The use of McQuillan's results in the logarithmic setting simplify the arguments used in [7] to obtain the algebraic degeneracy. Indeed, the authors need additional technical hypotheses to use the compact version of McQuillan's results.

Remark 6.14. In a sequel of this paper we will define and give some applications of higher order jet differentials. We will also develop an approach using stacks.

\section{Measure hyperbolicity AND ORBIFOLds of GENERAL TYPE}

7.1. Kobayashi-Ochiai's extension theorems. Let us recall some results about holomorphic mappings into orbifolds of general type.

In 19 it was established that a meromorphic map of maximal rank from a dense Zariski open subset $U$ of a complex manifold $V$ to a compact variety of general type extends meromorphically to $V$.

The main results of [29] can be formulated in the orbifold setting as follows.

Theorem 7.1 (Sakai). Let $(X, \Delta)$ be a smooth orbifold of general type where $X$ is a smooth projective manifold of dimension $n$. Then any orbifold morphism $f$ : $\mathbb{C}^{n} \rightarrow(X, \Delta)$ is degenerate; i.e., its Jacobian vanishes identically.

In [5] the following orbifold generalization of [19] was proved.

Theorem 7.2 (Campana). Let $V$ be a connected complex manifold, $Z$ a reduced divisor on $V$ and $U:=V \backslash Z$. Let $\varphi: U \rightarrow X$ be a meromorphic map with $X$ a projective manifold. Let $f: X \rightarrow Y$ be a fibration of general type and assume $\psi:=f \circ \varphi: U \rightarrow Y$ is of maximal rank (i.e., its differential is of maximal rank at some point).

Then $\psi$ extends meromorphically to $V$.

Here we would like to prove the following result, which generalizes both 29] and [5].

Theorem 7.3. Let $V$ be a connected complex manifold, $Z$ a reduced divisor on $V$ and $U:=V \backslash Z$. Let $\varphi: U \rightarrow(X, \Delta)$ be an orbifold morphism with $X$ a projective manifold. Let $f:(X, \Delta) \rightarrow Y$ be a fibration of general type and assume $\psi:=f \circ \varphi: U \rightarrow Y$ is of maximal rank. Then $\psi$ extends meromorphically to $V$.

An immediate corollary which provides new examples of special orbifolds is

Corollary 7.4. Let $(X, \Delta)$ be a smooth orbifold where $X$ is a smooth projective manifold of dimension $n$. Assume there exists a non-degenerate orbifold morphism $f: \mathbb{C}^{n} \rightarrow(X, \Delta)$. Then $(X, \Delta)$ is special.

The proof of the theorem will follow the same lines as [5] except that we have to take into account the orbifold structure on $X$.

Definition 7.5. Let $g:(X, \Delta) \rightarrow Y$ be a fibration with $(X, \Delta)$ and $Y$ smooth. $g$ is neat relative to $g^{\prime}:\left(X^{\prime}, \Delta^{\prime}\right) \rightarrow Y^{\prime}$ if there is a commutative diagram

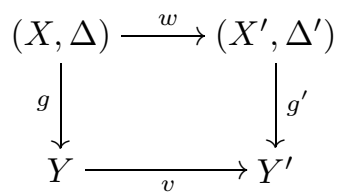


such that

(1) $w$ is an orbifold morphism, $v$ and $w$ are bimeromorphic and $w_{*}(\Delta)=\Delta^{\prime}$.

(2) Every $g$-exceptional divisor is $w$-exceptional.

We say that $g$ is strictly neat if moreover $(Y, \Delta(g))$ is smooth.

In this context we have (see [6])

Proposition 7.6. For every fibration $g^{\prime}:\left(X^{\prime}, \Delta^{\prime}\right) \rightarrow Y^{\prime}$, there exists $g:(X, \Delta) \rightarrow$ $Y$ strictly neat relative to $g^{\prime}$.

To deal with the orbifold structure on $X$ we will need the following proposition.

Proposition 7.7. Let $g:(X, \Delta) \rightarrow Y$ and $g^{\prime}:\left(X^{\prime}, \Delta^{\prime}\right) \rightarrow Y^{\prime}$ be fibrations with $\operatorname{dim} Y=p$ such that $g$ is neat relative to $g^{\prime}$.

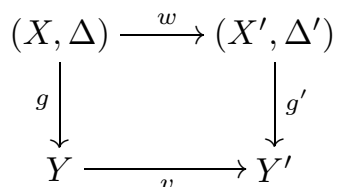

Let $m>0$ be an integer such that $m \Delta(g)$ is Cartier.

Then we have an injection of sheaves

$$
g^{*}\left(\mathscr{O}_{Y}\left(m\left(K_{Y}+\Delta(g)\right)\right)\right) \subset S^{m} \Omega_{(X, \Delta)}^{p} .
$$

Proof. Let $D \subset X$ be an irreducible divisor not $g$-exceptional and $x_{0} \in D$ a generic point with local coordinates $x=\left(x_{1}, \ldots, x_{n}\right)$ such that locally an equation of $D$ is $\left(x_{1}=0\right)$. Take local coordinates $\left(y_{1}, \ldots, y_{p}\right)$ near $g\left(x_{0}\right)$ such that $\Delta(g)$ has the equation

$$
y_{1}^{\left(1-\frac{1}{m_{1}}\right)}=0
$$

and $D$ is mapped locally to $y_{1}=0$. So, $g(x)=\left(x_{1}^{t_{1}}, \ldots, x_{p}\right)$ with $t_{1} \cdot m^{\prime} \geq m_{1}$ for $m^{\prime}=m_{\Delta}(E)$. Let

$$
\omega=\left(\frac{d y_{1} \wedge \cdots \wedge d y_{p}}{y_{1}^{\left(1-\frac{1}{m_{1}}\right)}}\right)^{\otimes m}
$$

be a local generator of $\mathscr{O}_{Y}\left(m\left(K_{Y}+\Delta(g)\right)\right)$. Then

$$
g^{*}(\omega)=x_{1}^{\frac{m t_{1}}{m_{1}}} \ldots x_{p}^{m}\left(\frac{d x_{1} \wedge \cdots \wedge d x_{p}}{x_{1} \ldots x_{p}}\right)^{\otimes m}
$$

up to a non-zero constant factor. Since $\frac{m t_{1}}{m_{1}} \geq\left\lceil\frac{m}{m^{\prime}}\right\rceil, g^{*}(\omega)$ is a local section of $S^{m, p}(X, \Delta)$. Therefore the injection is true outside $V \cup E(g)$, where $V$ is a subset of $X$ of codimension two or more contained above $\Delta(g)$ and $E(g)$ is the union of $g$ exceptional divisors. But then $w_{*}\left(g^{*}\left(\mathscr{O}_{Y}\left(m\left(K_{Y}+\Delta(g)\right)\right)\right)\right)$ injects into $S^{m} \Omega_{\left(X^{\prime}, \Delta^{\prime}\right)}^{p}$ over $X^{\prime}$ outside a codimension 2 or more analytic subset $Z$. Since these sheaves are locally free this injection extends through $Z$ by the Hartog extension theorem.

Now we give the proof of Theorem 7.3

Proof. We start with some reductions as in [18] and [5] to which we refer for details. We can reduce to the equidimensional case $\operatorname{dim} U=p$. By localizing we may assume that $V$ is a unit polydisc $D^{p}$ and $Z$ is a subpolydisc $\{0\} \times D^{p-1}$ so that $U=D^{*} \times D^{p-1}$. By Proposition 7.6 we can assume that $f$ is strictly neat. 
Since $K_{Y}+\Delta(f)$ is big, we have

$$
m\left(K_{Y}+\Delta(f)\right)=H+A,
$$

with $A$ very ample and $H$ effective. Let $\alpha \in H^{0}\left(Y, \mathscr{O}_{Y}(H)\right)$ be a non-zero section and $\delta \in H^{0}\left(Y, \mathscr{O}_{Y}(m \Delta)\right)$ a section vanishing exactly on $m \Delta$. Let $\sigma_{0}, \ldots, \sigma_{N}$ be a basis of $H^{0}(Y, A)$ and $s_{j}=\alpha \sigma_{j}$ and $t_{j}=\frac{s_{j}}{\delta}$. to prove that $\psi$ extends meromorphically to $V$, it suffices to show that the $\psi^{*}\left(s_{j}\right)$ extend to meromorphic sections of $m K_{V}$.

Then we define

$$
v:=\left(\sum_{j} i^{m p^{2}} t_{j} \wedge \overline{t_{j}}\right)^{\frac{1}{m}},
$$

which is a meromorphic pseudo-volume form on $Y$. From Proposition 7.7 we see that $f^{*}\left(t_{j}\right)$ is a holomorphic section of $S^{m, p}(X, \Delta)$ and therefore $w=\psi^{*}(v)$ is a pseudo-volume form on $U$. In the same way,

$$
\widetilde{w}=\left(\sum_{j} i^{m p^{2}} \psi^{*}\left(s_{j}\right) \wedge \overline{\psi^{*}\left(s_{j}\right)}\right)^{\frac{1}{m}}
$$

is a pseudo-volume form on $U$. Then a computation of the Ricci form gives

$$
\operatorname{Ricci}(\widetilde{w})=\operatorname{Ricci}(w)=\psi^{*}\left(-F^{*}(\Theta)\right),
$$

where $F: Y \rightarrow \mathbb{P}^{N}$ is the map defined by $A$ and $\Theta$ is the curvature form of the Fubini-Study metric. Therefore there exists $C>0$ such that the Ricci function

$$
K_{\widetilde{w}}:=-\frac{(-\operatorname{Ricci}(\widetilde{w}))^{p}}{\widetilde{w}} \leq-\frac{1}{C},
$$

and the Schwarz lemma for volume elements imply that

$$
\int_{U} \widetilde{w}<\infty
$$

Finally this implies that $\psi^{*}\left(s_{j}\right)$ extends to meromorphic sections of $m K_{V}$.

7.2. Orbifold measure hyperbolicity. In this section, we study measure hyperbolicity in the orbifold setting. We refer to [20, p. 109] for the basic facts on measure hyperbolicity and measure theory needed to state our results.

We consider Borel regular measures (i.e., they satisfy the property that the measure of a set is the inf of the measures of the open sets containing it). Let $f: X \rightarrow Y$ be holomorphic. It can be shown that if $A$ is a measurable set in $X$, then $f(A)$ is Borel measurable. Let $\mu, \nu$ be regular measures on $X$ and $Y$, respectively. $f$ is said to be measure decreasing if

$$
f^{*} \nu \leq \mu \text {, i.e., } \nu(f(A)) \leq \mu(A) \text { for all measurable } A \text {. }
$$

Instead of measurable $A$, it would suffice to take open sets $U$.

Definition 7.8. Let $(X, \Delta)$ be an orbifold.

(1) The orbifold Kobayashi pseudo-measure $\mu_{(X, \Delta)}$ on $(X, \Delta)$ is the largest measure on $X \backslash\lfloor\Delta\rfloor$ such that

$$
g^{*} \mu_{(X, \Delta)} \leqslant \mu_{P}
$$


for every orbifold morphism $g: \mathbb{D}^{n} \rightarrow(X, \Delta)$, where $\mu_{P}$ denotes the Poincaré measure on $\mathbb{D}^{n}$ induced by the Poincaré volume element

$$
\kappa_{n}=n ! \prod_{j=1}^{n} \frac{4}{\left(1-\left|z_{j}\right|^{2}\right)^{2}} \frac{i}{2} d z_{j} \wedge d \overline{z_{j}} .
$$

(2) The classical orbifold Kobayashi pseudo-measure $\mu_{(X, \Delta)}^{*}$ on $(X, \Delta)$ is the largest measure on $X \backslash\lfloor\Delta\rfloor$ such that

$$
g^{*} \mu_{(X, \Delta)} \leqslant \mu_{P}
$$

for every classical orbifold morphism $g: \mathbb{D}^{n} \rightarrow(X, \Delta)$.

Definition 7.9. Let $(X, \Delta)$ be an orbifold. Then $(X, \Delta)$ is said to be (classically) measure hyperbolic if $\left(\mu_{(X, \Delta)}^{*}(B)>0\right) \mu_{(X, \Delta)}(B)>0$ for every non-empty open subset $B \subset X \backslash\lfloor\Delta\rfloor$.

A theorem of Kobayashi and Ochiai (see [19]) proves that a projective complex manifold of general type is measure hyperbolic. In the logarithmic case, measure hyperbolicity of complements $X \backslash D$ of simple normal crossing divisors, such that $K_{X}+D$ is positive, was proved in [9]. Here we can generalize these results with

Theorem 7.10. Let $(X, \Delta)$ be a smooth projective orbifold of general type. Then $(X, \Delta)$ is measure hyperbolic.

Proof. This is essentially a consequence of [29]. Indeed, there, a volume form $\Psi$ is constructed on $X \backslash \operatorname{supp}(\Delta)$ such that $\int_{X \backslash \operatorname{supp}(\Delta)} \Psi<\infty$ and for every orbifold morphism $f: \mathbb{D}^{n} \rightarrow(X, \Delta), f^{*}(\Psi) \leq \kappa_{n}$. We recall its construction for the convenience of the reader. Let $A$ be an ample divisor on $X$. Then we can find an effective divisor $D$ in

$$
\left|m K_{(X, \Delta)}-A\right|
$$

for $m$ large enough. Let $\sigma$ be a section vanishing exactly on $D$. Let $\Delta=$ $\sum_{i}\left(1-\frac{1}{m_{i}}\right) Z_{i}$ with sections $s_{j}$ defining $Z_{j}$. We choose Hermitian metrics $h_{A}$ on $A, h_{j}$ on $Z_{j}$. Then we have a volume form on $X \backslash \operatorname{supp}(\Delta)$ defined, up to a constant $c$, locally by

$$
\Psi=c \frac{\|\sigma\|_{h_{A}^{-1}}^{\frac{2}{m}}}{\prod_{j=1}^{n}\left(\ln || s_{j} \|_{h_{j}}^{2}\right)^{2}\left|s_{j}\right|^{2\left(1-\frac{1}{m_{j}}\right)}} \prod_{j=1}^{n} \frac{i}{2 \pi} d z_{j} \wedge d \overline{z_{j}} .
$$

For every orbifold morphism $f: \mathbb{D}^{n} \rightarrow(X, \Delta), f^{*}(\Psi)$ is a singular volume element such that $\left(-\operatorname{Ricci}\left(f^{*}(\Psi)\right)\right)^{n} \geq f^{*}(\Psi)$ in the sense of currents. Therefore, using the Ahlfors-Schwarz lemma for volume elements, we obtain that $f^{*}(\Psi) \leq \kappa_{n}$. Finally, by definition, $\mu_{(X, \Delta)}$ is greater than the measure induced by $\Psi$ and $(X, \Delta)$ is measure hyperbolic.

\section{ACKNOWLEDGEMENTS}

We would like to thank Frédéric Campana and Michael McQuillan for their suggestions and their interest in this work. We also thank the referee for his remarks that improved the readability of this article. 


\section{REFERENCES}

[1] F.A. Bogomolov, Families of curves on a surface of general type, Soviet Math. Dokl. 236 (1977), 1294-1297. MR0457450 (56:15655)

[2] F.A. Bogomolov, Holomorphic tensors and vector bundles on projective manifolds, Math. USSR Izvestija 13 (1979), 499-555. MR0522939 (80j:14014)

[3] F.A. Bogomolov, Y. Tschinkel, Special elliptic fibrations, in Proc. Fano Conf., Torino (2003), ed. A. Conte, arXiv: math.AG/0303044. MR.2112577 (2005k:14017)

[4] M. Brunella, Courbes entières et feuilletages holomorphes, L'Enseignement Mathématique 45 (1999), 195-216. MR.1703368 (2000h:32046)

[5] F. Campana, Orbifolds, special varieties and classification theory, Ann. Inst. Fourier $\mathbf{5 4}$ (2004), 499-665. MR2097416 (2006c:14013)

[6] F. Campana, Orbifoldes spéciales et classification biméromorphe des variétés kählériennes compactes, arXiv:0705.0737.

[7] F. Campana, M. Păun, Variétés faiblement spéciales à courbes entières dégénérées, Compos. Math. 143 (2007), no. 1, 95-111. MR2295198(2008a:14012)

[8] F. Campana, J. Winkelmann, A Brody theorem for orbifolds, preprint 2006, arXiv: math/0604571.

[9] J. Carlson, P. Griffiths, A defect relation for equidimensional holomorphic mappings between algebraic varieties, Ann. of Math. (2) 95 (1972), 557-584. MR0311935 (47:497)

[10] X. Chen, On Algebraic Hyperbolicity of Log Varieties, Commun. Contemp. Math. 6 (2004), no. 4, 513-559. MR.2078413 (2005k:14089)

[11] J.-P. Demailly, Algebraic criteria for Kobayashi hyperbolic projective varieties and jet differentials, Proc. Sympos. Pure Math., vol.62, Amer. Math.Soc., Providence, RI, 1997, 285-360. MR.1492539 (99b:32037)

[12] G. Dethloff, S. Lu, Logarithmic jet bundles and applications, Osaka J. of Math. 38, 2001, 185-237. MR.1824906 (2002f:32032)

[13] J. El Goul, Logarithmic jets and hyperbolicity, Osaka J. Math. 40 (2003), 469-491. MR1988702 (2004h:32026)

[14] H. M. Farkas, I. Kra, Riemann Surfaces, Springer-Verlag, New-York, 1980, second edition. MR583745 (82c:30067)

[15] A. Ghigi, J. Kollár, Kähler-Einstein metrics on orbifolds and Einstein metrics on spheres, Comment. Math. Helv. 82 (2007), 877-902. MR2341843(2008j:32027)

[16] M. Green, P. Griffiths, Two applications of algebraic geometry to entire holomorphic mappings, The Chern Symposium 1979, Proc. Inter. Sympos. Berkeley, CA, 1979, Springer-Verlag, New-York, 1980, 41-74. MR609557 (82h:32026)

[17] J.-P. Jouanolou, Hypersurfaces solutions d'une équation de Pfaff analytique, Math. Ann. 232 (1978), 239-245. MR0481129 (58:1274)

[18] S. Kobayashi, Hyperbolic complex spaces, Springer-Verlag, Berlin, 1998. MR.1635983 (99m:32026)

[19] S. Kobayashi, T. Ochiai, Meromorphic mappings into compact complex spaces of general type, Invent. Math., 31 (1975), 7-16. MR0402127 (53:5948)

[20] S. Lang, Introduction to complex hyperbolic spaces, Springer-Verlag, New York, 1987. MR886677 (88f:32065)

[21] M. McQuillan, Diophantine approximations and foliations, Publ. IHES 87 (1998), 121-174. MR.1659270 (99m:32028)

[22] M. McQuillan, Noncommutative Mori theory, preprint IHES (2000).

[23] M. McQuillan, Bloch hyperbolicity, preprint IHES (2001).

[24] M. McQuillan, Rational criteria for hyperbolicity, Book preprint.

[25] R. Nevanlinna, Analytic functions, Berlin-Heidelberg-New York, Springer-Verlag, 1970. MR0279280 (43:5003)

[26] E.I. Nochka, On the theory of meromorphic functions, Soviet Math. Dokl. 27 (2) (1983), 377-381. MR0701289 (85i:32038)

[27] G. Pacienza, E. Rousseau, On the logarithmic Kobayashi conjecture, J. Reine Angew. Math. 611 (2007), 221-235. MR2361090 (2008j:14084)

[28] E. Rousseau, Hyperbolicité du complémentaire d'une courbe: le cas de deux composantes, CRAS Ser. I 336 (2003), 635-640. MR1988123 (2004f:14046) 
[29] F. Sakai, Degeneracy of holomorphic maps with ramification, Invent. Math. 26 (1974), 213229. MR0355118 (50:7595)

[30] A. Seidenberg, Reduction of singularities of the differential equation $A d y=B d x$, Amer. J. Math. 90 (1968) 248-269. MR0220710 (36:3762)

[31] B.V. Shabat, Distribution of values of holomorphic mappings, Translated from the Russian by J. R. King. Translation edited by Lev J. Leifman. Translations of Mathematical Monographs, 61. American Mathematical Society, Providence, RI, 1985. MR.807367 (86k:32023)

[32] Y.T. Siu, A proof of the generalized Schwarz lemma using the logarithmic derivative lemma. Private communication to J.-P. Demailly, Journal de la SMF (1997).

[33] P. Vojta, On the ABC conjecture and Diophantine approximation by rational points, Amer. J. Math. 122 (2000), no. 4, 843-872. MR1771576 (2001i:11094)

[34] P.-M. Wong, Nevanlinna theory for holomorphic curves in projective varieties, preprint (1999).

Département de Mathématiques, iRma, Université Louis Pasteur, 7, Rue René Descartes, 67084 Strasbourg Cedex, France

E-mail address: rousseau@math.u-strasbg.fr 